\title{
Caracterización del cielo de Copán del 400 al 900 d. C.
}

Nohemy Lizeth Rivera Gutiérrez

\section{Resumen}

La ocurrencia de eventos astronómicos, en muchos casos, es un suceso que se puede predecir, existiendo herramientas y métodos para pronosticar diversos eventos astronómicos que suceden y/o que han sucedido en el pasado. Particularmente los mayas conocían el movimiento de los astros con gran detalle, la ocurrencia de estos eventos pudo haber sido registrado por los mayas en Copán, por lo tanto, se pretende aportar información que pueda apoyar al trabajo de búsqueda e interpretación del legado astronómico dejado por los mayas en Copán. El objetivo consiste en identificar los eventos astronómicos relevantes, visibles a simple vista y eventuales, en el sitio de Copán durante el período 400 d.C. al 900 d.C., mediante la identificación de la ocurrencia de eclipses solares y lunares. El período de tiempo seleccionado del estudio coincide con el desarrollo de la Dinastía maya de Copán, del 400 al 900 d.C. La investigación es de tipo descriptivo y analítica, las variables en análisis incluyen el tipo de eclipse, fechas de ocurrencia de eclipses, tiempos de duración y magnitud observable del eclipse. Para la recolección de la información se han utilizado las bases de datos del Goddard Space Flight Center de la NASA para el período del estudio y en la ubicación geográfica de Copán. A partir del estudio se han identificado los datos para 160 eclipses solares y 236 eclipses lunares, incorporando las fechas julianas y clasificando en función de la Cuenta Larga correspondiente, se definió que durante este período no ocurrieron eclipses solares totales en esta zona y que en el período de Durante el período de Waxaklaju'n U B'aah K'awiil se produjo un eclipse solar anular, 3 eclipses solares parciales con más del $75 \%$ de magnitud observable y 19 eclipses lunares totales.

Palabras claves: Eclipses solares, eclipses lunares, Copán. 


\section{Abstract}

The occurrence of astronomical events, in many cases, can be predicted, existing tools and methods to predict various astronomical events that occur and / or that have happened in the past. Particularly, the Maya recognized the movement of the stars in great detail, the occurrence of these events could have been recorded by the Maya in Copan, therefore, this information can support the work of research and interpretation of astronomical legacy of the Maya of Copan. The aim is to identify the relevant astronomical events, visible to the naked eye, at the site of Copan during the period $400 \mathrm{AD}$ to $900 \mathrm{AD}$, by identification of solar and lunar eclipses. The selected time period coincides with the development of the Mayan Copan Dynasty. The research is descriptive and analytical type, the variables include the type of eclipse, dates of occurrence of eclipses, time duration and magnitude of the eclipses. For the collection of information,it have been used Goddard Space Flight Center of NASA databases for the geographical location of Copan. From the data, they were identified 160 solar eclipses and 236 lunar eclipses, incorporating the Julian dates and classified according to the corresponding Long Count.During this period, no total solar eclipses occurred in this area and during the period of Waxaklaju'n UB'aah K'awiil there werethree partial solar eclipses with more than $75 \%$ of observable magnitude, an annular solar eclipse and nineteen total lunar eclipses.

Keywords: Solar eclipses, lunar eclipses, Copan.

Nohemy Lizeth Rivera Gutiérrez, Departamento de Arqueoastronomía y Astronomía Cultural, Universidad Nacional Autónoma de Honduras 


\section{Introducción}

La ocurrencia de eventos astronómicos, en muchos casos, es un suceso que se puede predecir. La astronomía de posición actual tiene herramientas para predecir con exactitud muchos de los eventos astronómicos que suceden. Pero también, se conoce suficiente para detallar los eventos que han sucedido en el pasado. El registro de la ocurrencia de los eventos astronómicos ha sido efectuado por múltiples culturas. Los mayas conocían el movimiento de los astros con gran detalle, llegando hasta dejar registros extensos de los movimientos de Venus, la Luna y Marte en los códices (Aveni, 2005). Por lo que conviene analizar los eventos celestes ocurridos en Copán en el periodo del 400 al $900 \mathrm{~d}$. C. utilizando las herramientas digitales actuales para tener la posibilidad de identificar sus registros en Copán.

La Arqueoastronomía, en parte, busca identificar las prácticas y usos de los conocimientos astronómicos de las culturas antiguas. La identificación de los eventos celestes que se dieron en Copán durante el período de desarrollo de la ciudad puede aportar conocimientos para los estudios que se realizan en el sitio arqueológico, especialmente los eventos astronómicos observables a simple vista y eventuales durante el período 400 d.C. al 900 d.C., en la ubicación geográfica de $14^{\circ} 56^{\prime} \mathrm{N}$ y $88^{\circ} 51^{\prime} \mathrm{W}$. Existen diversos monumentos erigidos en Copán durante esa época,contienen inscripciones, éstas pueden contener información sobre eventos astronómicos registrados.

\section{Los eclipses de Sol}

Las culturas antiguas se percataron de los movimientos de los astros en el cielo, diversas culturas dejaron registros de esos conocimientos (Ruggles, 2005). Los mayas, particularmente, en los códices, han dejado registros relacionados a Venus, Marte y eclipses (Aveni, 2005).

Los eclipses de Sol son eventos astronómicos recurrentes, que pueden provocar gran interés y curiosidad. Este fenómeno en la bóveda celeste, se produce con la participación del Sol y la Luna, cuyos tamaños aparentes en el cielo son similares, permitiendo así que en determinados puntos de su recorrido la Luna cubra al Sol y su luz. Para un observador en la superficie de la Tierra, se visualiza que el disco solar, poco a poco, está siendo cubierto por una sombra circular oscura, esto es lo que nosotros llamamos eclipse de Sol. Esto permite que aunque sea de día, el ambiente se oscurezca y se puedan observar estrellas brillantes y planetas. Este fenómeno solo ocurre cuando la luna está en su fase nueva. Este eclipse puede tener diferentes disposiciones, puede ser un eclipse parcial (donde solo llega a cubrirse parte del disco solar), eclipse total (poco a poco llega a cubrirse toda la superficie del disco solar) y eclipse anular (cuando es cubierto todo el centro solar pero queda un anillo brillante del disco solar alrededor de la sombra oscura). 
Sin embargo, la posibilidad de observar un eclipse total o anular, en cada vez que ocurre un fenómeno de este tipo, solo es posible en un franja angosta de la Tierra, en el resto de la superficie del recorrido del eclipse solo se observan diferentes grados de parcialidad, es decir que el Sol solo se cubre parcialmente. La zona de la Tierra donde se observa el eclipse total se conoce como banda de totalidad, suele medir entre 40 y $100 \mathrm{~km}$ de anchura y varios miles de kilómetros de longitud, que se debe al movimiento de rotación de la Tierra y de la Luna. Esta zona tiene un inicio, que es el punto de la Tierra donde se observa primero la totalidad y luego se va trasladando. En esa zona, la luz del Sol llega a desaparecer totalmente, dependiendo cada caso, durante un máximo de $7 \mathrm{~min} 31 \mathrm{~s}$, si es un eclipse total, pero en general dura alrededor de 3 minutos de duración(William, 2006). Por ejemplo, puede ser que en una ciudad particular se observe un eclipse total, pero en otra ciudad cercana o de la misma región solo sea observado un eclipse parcial, por lo que los datos de los eclipses solares no aplican por igual a todas las regiones.

En la imagen 1 se muestra un mapa de la Tierra donde se grafica las zonas donde se observó el eclipse de Sol del 21 de octubre de 450 d.C., en el centro del mapa se observan dos líneas paralelas, muy cercanas entre sí, estas líneas definen la zona en la Tierra donde se observó el eclipse total de Sol, donde la superficie visible del Sol fue ocultada totalmente por la Luna. En las regiones que están incluidas entre las otras líneas paralelas a la zona de totalidad se observó un eclipse parcial de Sol, dicha parcialidad va disminuyendo a medida que la región está más alejada del área de la totalidad. En el resto del planeta, que no está incluido en la zona marcada no se observó el eclipse. Cada eclipse transita diferentes zonas de la superficie. En este caso, para este eclipse, en la zona de Copán el eclipse observable fue parcial, dado que no coincidió con la zona de totalidad, pero la parcialidad fue mayor al $50 \%$, es decir que más del $50 \%$ de la superficie visible del Sol fue cubierta por la Luna. 


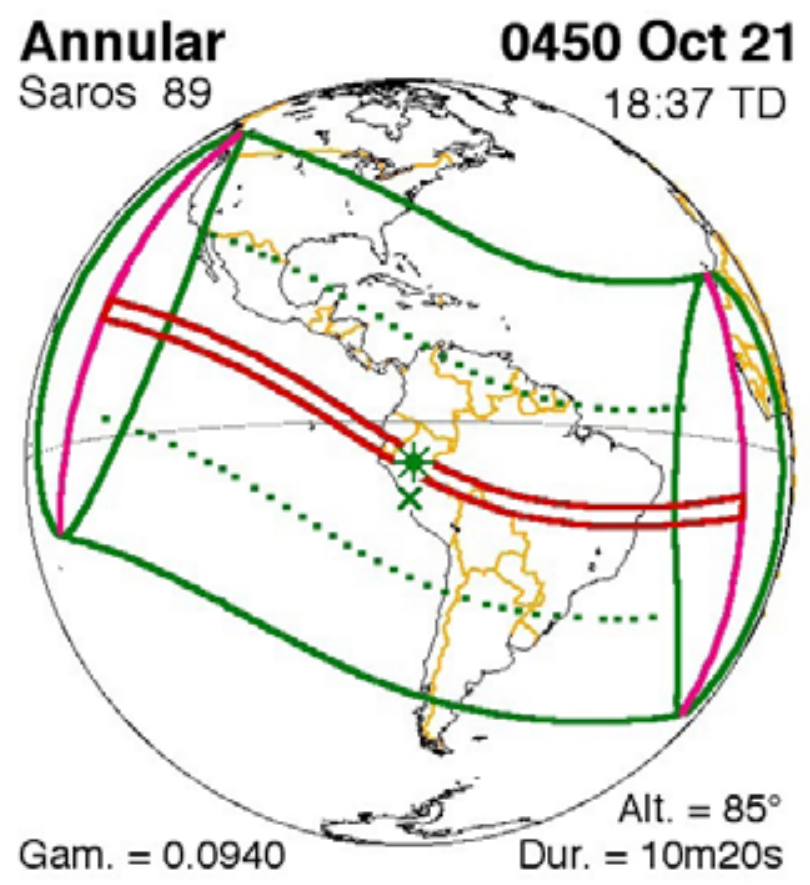

Five Millennium Canon of Solar Eclipses (Espenak \& Meeus)

Imagen 1: Mapa del Eclipse solar del 21 de octubre de 405 d.C.

(Espenak, Five millennium catalog of solareclipses, 2009)

En relación a la ocurrencia de los eclipses de Sol, se le conoce con el nombre de período Saros a un lapso de 6,585 días, es decir, alrededor de 18 años y 10 u 11 días (según haya años bisiestos en este período) al cabo de los cuales se repiten aproximadamente los mismos eclipses con características similares (William, 2006).

\section{Los eclipses de Luna}

Por otra parte, los eclipses de luna se producen en el cielo nocturno, coincidiendo con la Luna Llena, es decir, una luna que está en su máximo brillo. La Luna inicia a oscurecerse, de forma tenue con una sombra circular que poco a poco va cubriendo a este astro, esta sombra se le llama penumbra, pero que no es muy evidente a la observación a simple vista. Seguidamente, la Luna va siendo cubierta por una segunda sombra circular, llamada umbra, más oscura pero que le va dando una apariencia a la Luna Llena de color rojizo. Si la Luna llega a cubrirse 
totalmente por la umbra será un eclipse total de Luna, y en el caso que se trate de un eclipse parcial solo la cubrirá parcialmente. Puede suceder que la luna solo sea cubierta por la sombra penumbral, por lo que ese tipo de eclipse se llama eclipse lunar penumbral, pero esta sombra es poco apreciable a la visión humana.

Los eclipses de luna son más frecuentes que los eclipses de Sol, cada año ocurren de 1 o 2 eclipses de Luna, pero visibles desde todo el hemisferio nocturno de la Tierra y, por lo tanto, resultan más fáciles de observar. Asimismo, la duración de los eclipses lunares es de mayor tiempo, con un promedio de 3 horas en la fase umbral (Martínez, Miralles, Marco, \& Galadí-Enriquez, 2005).

\section{Los Mayas y los eclipses}

El Sol y la Luna eran elementos importantes de la cosmogonía maya. Se han estudiado las diversas formas que los grupos culturales de la región mesoamericana han relacionado al Sol y a la Luna, como padre y madre, como abuelo y abuela, madre e hija, como esposos (Thompson, 2006). Por lo que resulta relevante cada astro por separado como los eventos astronómicos donde se relacionan, por ejemplo en los eclipses. El Códice de Dresde presenta unas tablas de números y glifos (página 51-58 del Códice), estos registros han sido estudiados por diversos autores, asociándolos unos a datos lunares y otros autores a registros de eclipses lunares (Ruggles, 2005). Sin embargo, queda claro su relación a los movimientos sinódicos de la luna y el Sol, dado que en las tablas se reproduce (contabiliza) el período de Saros de 6,585 días.

Según Rice (2004), los antiguos mesoamericanos comenzaron a elaborar registros permanentes de eventos astronómicos alrededor de la primera mitad del primer milenio después de Cristo, posiblemente casi simultáneamente en la región del Golfo y en Oaxaca. Esta astronomía predictiva que practicaban los mesoamericanos tiene que basarse en un gran cúmulo de registros observacionales por siglos, permitiendo el reconocimiento de periodicidades.

Por otra parte, en cuanto al significado atribuido a estos eventos, se plantea que la ocurrencia de un eclipse se asociaba a eventos catastróficos o desafortunados (Ruggles, 2005).Las explicaciones populares de los eclipses de sol y de luna varían considerablemente entre los pueblos mayas, creencias de que los eclipses se deben a peleas conyugales entre el Sol y la Luna; que los eclipses lunares son causados por un jaguar, una variedad de hormigas o algunos demonios (Thompson, 2006) o que implicaba que la Luna era atacada, mordida o comida, por lo tanto se enfermaba o estaba muriendo (Ruggles, 2005). Asimismo, lo relacionaban a enfermedades que pudieran sufrir las personas. Una costumbre que todavía existe consiste en hacer mucho ruido para distraer la atención del agresor y salvar al Sol o la Luna (Thompson, 2006). Estos aspectos demuestran que entre los mayas, tanto el registro como la significación de estos eventos resultan relevantes. 


\section{Metodología}

La presente investigación es de tipo descriptivo, transversal, analítica, no experimental. Para la recolección de la información se utilizaron las bases de datos de eclipses solares y lunares del sitio web del Goddard Space Flight Cente de la National Aeronautics and Space Administration (NASA) para el período del 400 al 926 d.C. en la ubicación geográfica de Copán ( $14^{\circ} 56^{\prime} \mathrm{N}$ y $\left.88^{\circ} 51^{\prime} \mathrm{W}\right)$. A partir de los datos de eclipses solares y lunares, se clasificaron por tipo de eclipse solar (total, anular o parcial) y tipo de eclipse lunar (penumbral, total y parcial), identificando la ocurrencia de eclipses cada tipo y cuantificando la ocurrencia de cada tipo de eclipse. Asimismo, se analizaron los datos en función de su duración, magnitud del eclipse $^{1}$ y tipo de eclipse, diferenciándolos en función de la fecha de Cuenta Larga, bajo la correlación astronómica 584285. Los eclipses anulares de Sol se relacionaron a los gobernantes que regían en Copán durante la ocurrencia del fenómeno.

\section{Resultados}

\section{Eclipses de Sol}

Para el período estudiado se identificaron 160 eclipses de Sol, de los cuales el $3 \%$ fueron eclipses anulares y el $97 \%$ fueron eclipses parciales (Tabla 1 ).

\begin{tabular}{|c|c|c|}
\hline \multicolumn{3}{|c|}{ Tabla 1: Eclipses solares en Copán del 400 al 900 d.C. } \\
\hline Tipos de eclipses & Cantidad & Porcentaje \\
\hline Eclipse Anular & 5 & $3 \%$ \\
\hline Eclipse Parcial & 155 & $97 \%$ \\
\hline Total & 160 & $100 \%$ \\
\hline
\end{tabular}

Tabla 1: Eclipses solares en Copán del 400 al 900 d.C.

Según la magnitud observable de los eclipses parciales (Tabla 2), se realizó una distribución de la frecuencia, resultando un 30\% de los eclipses con magnitudes menores al $25 \%$, un $28 \%$ de los eclipses con magnitudes entre $25 \%$ a $50 \%$, un $22 \%$ con magnitudes entre $50 \%$ a $75 \%$ y un $20 \%$ de los eclipses con magnitudes superiores a $75 \%$, se puede observar un descenso en la cantidad de eclipses en función que aumenta la magnitud del eclipse solar parcial.

\footnotetext{
${ }^{1}$ La magnitud observable para los eclipses de Sol está definida como la fracción del diámetro del Sol inmerso en la sombra de la Luna y la magnitud observable para los eclipses de Luna está definida como la fracción del diámetro de la Luna cubierto en la sombra de la Tierra.
} 


\begin{tabular}{|c|c|c|}
\hline \multicolumn{3}{|c|}{ Tabla 2: Eclipses solares parciales por magnitud observable } \\
$\begin{array}{c}\text { Magnitud } \\
\text { observable }\end{array}$ & $\begin{array}{c}\text { Número de } \\
\text { eclipses }\end{array}$ & Porcentaje \\
\hline $0-25 \%$ & 46 & $30 \%$ \\
\hline $25 \%-50 \%$ & 44 & $28 \%$ \\
\hline $50 \%-75 \%$ & 34 & $22 \%$ \\
\hline $75 \%-100 \%$ & 31 & $20 \%$ \\
\hline Total & 155 & $100 \%$ \\
\hline
\end{tabular}

Tabla 2: Eclipses solares parciales por magnitud observable

Un $42 \%$ de los eclipses (65 eclipses) mostraron una magnitud por encima del $50 \%$, es decir, más de la mitad del diámetro del disco solar cubierto por la Luna. Estos eclipses con magnitudes mayores son notablemente apreciables.

En el gráfico 1 se describen los eclipses anulares ocurridos en el período de estudio (5 eclipses anulares), se presentan los tiempos en minutos del máximo del eclipse (tiempo durante magnitud máxima en eclipses totales y anulares). Los tiempos de los eclipses anulares de Sol van desde 2 minutos 15 segundos hasta 9 minutos 11 segundos. El eclipse con el tiempo mayor durante la magnitud máxima del eclipse sucedió el 19 de enero del 744. 


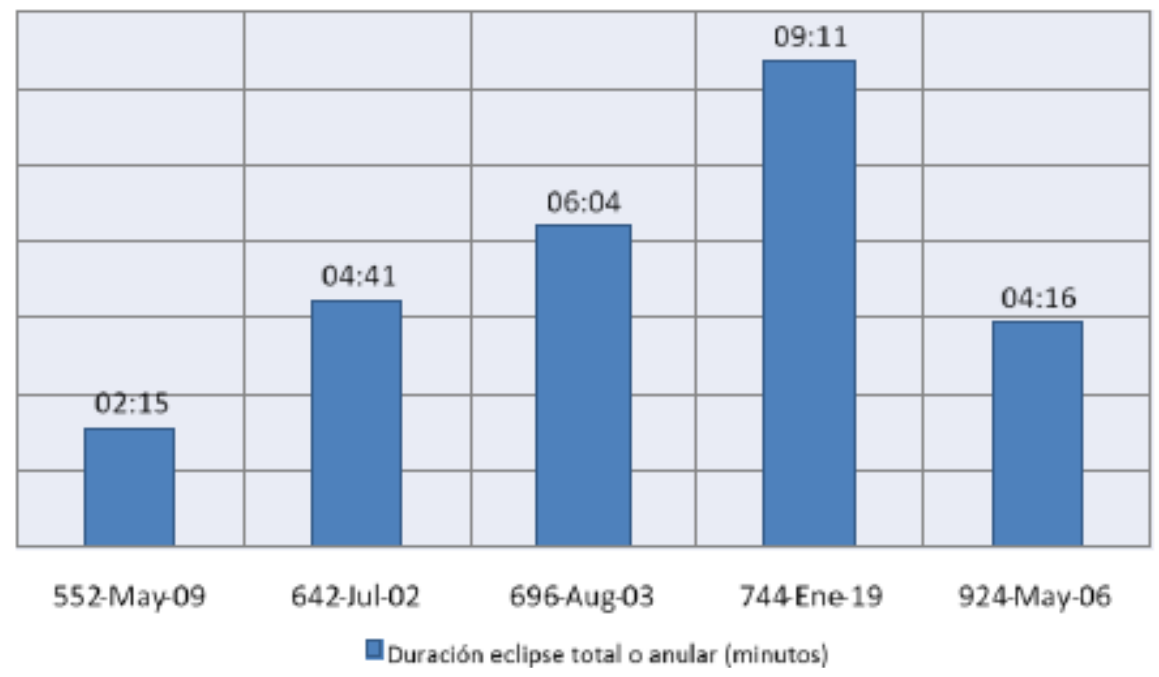

Gráfico 1: Eclipses solares anulares por duración y fecha, en Copán (duración en minutos) Elaboración propia. Fuente: (Espenak, Five millennium catalog of solar eclipses, 2009)

En el gráfico 2 se muestran los datos de los cinco eclipses anulares de Sol, específicamente se describen las magnitudes observables de estos eclipses pero el eclipse de magnitud observable. Dado que en los eclipses anulares queda visible la sección perimetral del disco solar, las magnitudes observables no llegan al $100 \%$, pero si sobrepasan el $90 \%$. En este caso, los eclipses presentan magnitudes que van desde el $92.2 \%$ hasta el $94.5 \%$. El eclipse con mayor magnitud sucedió el del 6 de mayo del 924 . Mientras que el eclipse de mayor duración durante el máximo, el eclipse del año 744 , solo presentó una magnitud del $92.2 \%$. 


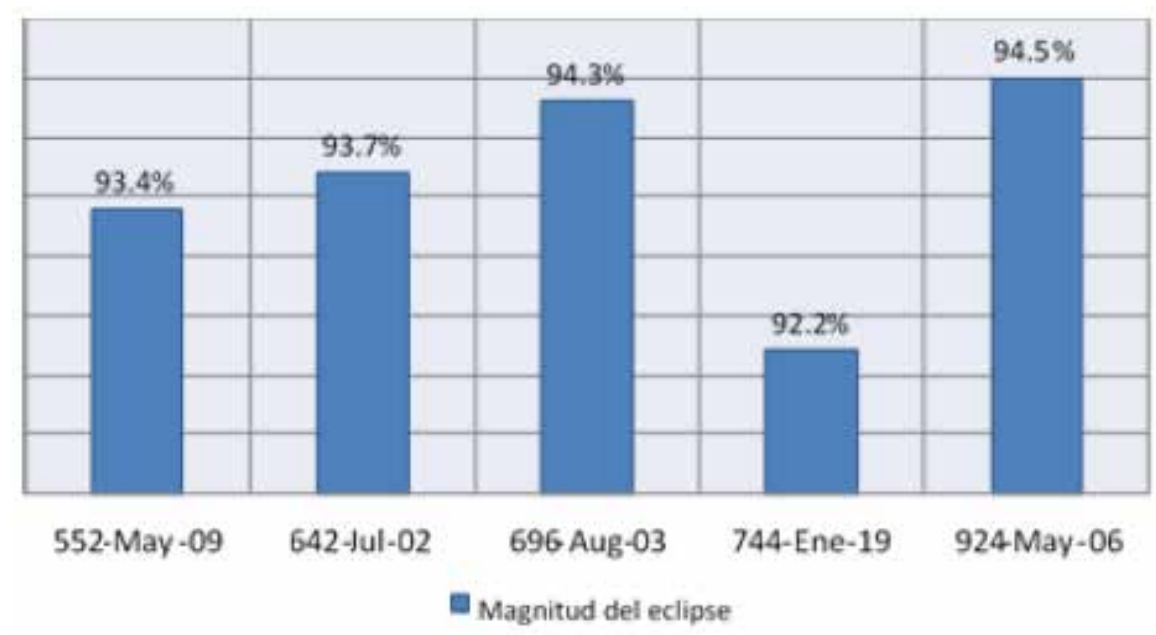

Gráfico 2: Eclipses solares anulares por magnitud y fecha, en Copán Elaboración propia. Fuente: (Espenak, Five millennium catalog of solar eclipses, 2009)

En la tabla 3 se presentan diversos datos de los eclipses solares anulares, se describen las fechas julianas, fecha de Cuenta Larga, Tzolkin y Haab, las horas de ocurrencia de la parcialidad y máximo de cada eclipse, la magnitud observable, la duración de su máximo, la duración total (incluyendo desde el inicio de la parcialidad hasta su final) y se agrega el nombre del gobernante durante ese tiempo. Por ejemplo, el eclipse sucedido el 19 de enero del 744, tiene una fecha de 9.15.12.10.17 4 K'aban 10 K'umk'u. Este eclipse inició temprano en la mañana, (8:45 a.m.), la fase anular se produjo a media mañana (de 10:34 - 10:43 a.m.) y finalizó después del mediodía solar (12:41 p.m.), con una duración total del eclipse de 3 horas y 56 minutos. 


\begin{tabular}{|c|c|c|c|c|c|}
\hline $\begin{array}{l}\text { Fecha calendario ju iano del } \\
\text { eclipse }\end{array}$ & 9-may-552 & 2-jul-642 & 3-ago-696 & 19-ene-744 & 6-may-924 \\
\hline \multirow{3}{*}{$\begin{array}{c}\text { Fecha Cuenta larga* } \\
\text { Tzolkin } \\
\text { Haab } \\
\end{array}$} & 9.5 .18 .2 .0 & 9.10 .9 .10 .6 & 9.13 .4 .8 .2 & 9.15 .12 .10 .17 & 10.4 .15 .9 .10 \\
\hline & 5 Ajaw & 2 Kimi' & $13 \mathrm{~K}$ 'an & 4 K'aban & $12 \mathrm{Ok}$ \\
\hline & $8 \mathrm{Sip}$ & 4 Yaxkin $^{\top}$ & 12 Chén & $10 \mathrm{~K}^{\prime} u \mathrm{mk}^{\prime} u$ & 18 Yaxk'in \\
\hline \multirow{3}{*}{$\begin{array}{l}\text { Inicio eclipse parcial } \\
\text { Inicio eclipse anular } \\
\text { Hora máximo eclipse }\end{array}$} & $05: 28$ & $15: 57$ & $08: 34$ & $08: 45$ & $08: 25$ \\
\hline & $05: 41$ & $17: 14$ & $10: 23$ & 10:34 & 09:56 \\
\hline & $05: 42$ & $17: 16$ & $10: 26$ & 10:39 & $09: 59$ \\
\hline \multirow{3}{*}{$\begin{array}{l}\text { Final eclipse anular } \\
\text { Final eclipse parcial } \\
\text { Magnitud eclipse }\end{array}$} & $05: 43$ & $17: 19$ & $10: 29$ & $10-43$ & $10: 01$ \\
\hline & $06: 47$ & $18: 23$ & $12: 34$ & $12-41$ & $11: 49$ \\
\hline & $93.40 \%$ & $93.70 \%$ & $94.30 \%$ & $92.20 \%$ & $94.50 \%$ \\
\hline \multirow{2}{*}{$\begin{array}{c}\text { Duración del eclipse } \\
\text { (hr) } \\
\text { Duración del máximo del } \\
\text { eclipse (minutos) }\end{array}$} & $01 \mathrm{~h} 19 \mathrm{~m}$ & $02 \mathrm{~h} 26 \mathrm{~m}$ & $04 \mathrm{~h} 00 \mathrm{~m}$ & $03 \mathrm{~h} 56 \mathrm{~m}$ & $03 \mathrm{~h} 24 \mathrm{~m}$ \\
\hline & $02: 15$ & $04: 41$ & $06: 04$ & 09:11 & $04: 16$ \\
\hline Gobemante & Sak Lu & 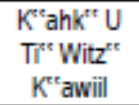 & $\begin{array}{c}\text { Waxaklajuu n } \\
\text { Ub'aah } \\
\text { K'awiil }\end{array}$ & $\begin{array}{l}\text { Kák' Joplaj Chan } \\
\text { Káwiil }\end{array}$ & - \\
\hline
\end{tabular}

Tabla 3: Eclipses Anulares de Sol del 400 al 926 d.C. en Copán

A partir de la tabla 3, se puede conocer que los eclipses anulares que sucedieron en Copán en el período de estudio, fueron eclipses que pudieron ser observados completos, es decir, dadas las horas de inicio y final de la parcialidad, sucedieron durante el día para la ubicación de Copán. Por otra parte, en relación a la duración del eclipse completo, el eclipse anular más extendido sucedió el 3 de agosto de 696, durante el reinado de Waxaklajuun Ub'aah K'awiil y el más corto el eclipse del 9 de mayo de 552.

La dirección de Copán durante estas fechas de ocurrencia de estos eclipses se tiene que para el eclipse del 9 mayo del 552, se menciona que reinaba el noveno gobernante de Copán, Sak Lu; para el 2 de julio del 642 corresponde al período del doceavo gobernante K'ahk' U Ti' Witz' K'awiil, y en el eclipse del 3 de agosto del 696 sucedió durante el mandato de Waxaklajuun Ub'aah K'awiil. El siguiente sucedió el 19 de enero del 744 en el período de Kák' Joplaj Chan Káwiil y para el 6 de mayo del 924 no se registra algún gobernante definido (Tabla 3).

Asimismo, para los eclipses parciales se observa en la Tabla 4, la fecha juliana para cada eclipse parcial, la hora de la máxima parcialidad del eclipse, la magnitud observable (porcentaje del diámetro solar cubierto) y la duración del eclipse en horas, minutos y segundos. Para algunos de los eclipses se da la situación que el Sol se levanta por el horizonte, es decir, amanece, pero el eclipse ya ha iniciado (salida del Sol eclipsado), o por el contrario, se oculta por la tarde y el eclipse no ha finalizado (puesta de Sol eclipsado), información que aparece en la columna de observaciones. 
A partir de esta tabla se puede detectar que cada katún presenta de 2 hasta 10 eclipses por dicho período. Durante el primer Katun del noveno Baktun ocurrieron 10 eclipses con magnitudes que van desde el $16 \%$ hasta un eclipse que llega a una magnitud del $93 \%$. En este período se observa que el Sol durante el día 2 de enero de 447 se oculta eclipsado. A continuación se presentan los eclipses que ocurrieron en Copán desde el siglo $\mathrm{V}$ al siglo $\mathrm{X}$, pero clasificados según el Katun de la Cuenta Larga en el que ocurrieron:

\begin{tabular}{|c|c|c|c|c|}
\hline Fecha juliana & $\begin{array}{l}\text { Máximo del } \\
\text { eclipse } \\
\text { (Hora del eclipse) }\end{array}$ & $\begin{array}{l}\text { Magnitud } \\
\text { Eclipse } \\
\text { Observable }\end{array}$ & $\begin{array}{l}\text { Duración del } \\
\text { eclipse } \\
\text { observable (Horas: } \\
\text { minutos: segundos) }\end{array}$ & Observaciones \\
\hline \multicolumn{5}{|c|}{ Cuenta larga: $8 \cdot 18 \cdot 0.0 .0$} \\
\hline $404-A b r-25$ & $06: 13$ & $68 \%$ & $01: 37: 07$ & salida de Sol eclipsado \\
\hline 410-Jun-18 & 05:24 & $36 \%$ & $00: 31: 21$ & salida de Sol eclipsado \\
\hline 411-Dic-01 & 13:53 & $36 \%$ & $02: 24: 03$ & \\
\hline 413-Abr-16 & $05: 41$ & $16 \%$ & $00: 15: 27$ & salida de Sol eclipsado \\
\hline \multicolumn{5}{|c|}{ Cuenta larga: $8 \cdot 19 \cdot 0.0 .0$} \\
\hline 417-Jul-29 & $13: 04$ & $17 \%$ & 01:58:59 & \\
\hline $420-$ Nov-21 & $11: 57$ & $86 \%$ & $03: 03: 36$ & \\
\hline 424-Mar-16 & $15: 43$ & $32 \%$ & 01:53:20 & \\
\hline 428-Jun-28 & $14: 52$ & $56 \%$ & $02: 50: 16$ & \\
\hline \multicolumn{5}{|c|}{ Cuenta larga: $9 \cdot 0 \cdot 0.0 .0$} \\
\hline 436-Jul-29 & $12: 52$ & $32 \%$ & $02: 40: 22$ & \\
\hline 439-May-28 & $14: 54$ & $92 \%$ & 03:19:01 & \\
\hline 439-Nov-22 & $08: 18$ & $16 \%$ & $01: 41: 50$ & \\
\hline 443-Mar-17 & 09:07 & $27 \%$ & $02: 19: 44$ & \\
\hline 443-Sep-09 & $12: 24$ & $25 \%$ & $02: 12: 13$ & \\
\hline 447-Ene-02 & $16: 58$ & $90 \%$ & $01: 44: 51$ & puesta de Sol eclipsado \\
\hline $450-A b r-27$ & $14: 08$ & $93 \%$ & $02: 48: 49$ & \\
\hline $450-0 c t-21$ & 09:40 & $54 \%$ & $03: 25: 59$ & \\
\hline $451-0 c t-10$ & $13: 23$ & $30 \%$ & 02:50:09 & \\
\hline $453-F e b-24$ & $07: 54$ & $83 \%$ & $02: 57: 25$ & \\
\hline
\end{tabular}

Tabla 4: Eclipses parciales de Sol del 400 al 926 d.C. en Copán 


\begin{tabular}{|c|c|c|c|c|}
\hline Fecha juliana & $\begin{array}{c}\text { Máximo del eclipse } \\
\text { (Hora del eclipse) }\end{array}$ & $\begin{array}{l}\text { Magnitud } \\
\text { Eclipse } \\
\text { Observable }\end{array}$ & $\begin{array}{l}\text { Duración del edipse } \\
\text { observable (Horas: } \\
\text { minutos: segundos) }\end{array}$ & Observaciones \\
\hline \multicolumn{5}{|c|}{ Cuenta larga: 9.1.0.0.0 } \\
\hline 472-Feb-24 & $18: 02$ & $31 \%$ & $00: 31: 40$ & puesta de Sol eclipsado \\
\hline 474-Dic-24 & $16: 21$ & $89 \%$ & $02: 17: 29$ & \\
\hline \multicolumn{5}{|c|}{ Cuenta larga: $9 \cdot 2 \cdot 0 \cdot 0.0$} \\
\hline $478-A b r-18$ & $14: 41$ & $8 \%$ & $01: 27: 13$ & \\
\hline 483-Ene-24 & $07: 23$ & $7 \%$ & 01:11:06 & \\
\hline 483-Jul-20 & $10: 54$ & $70 \%$ & 03:50:47 & \\
\hline 490-Ago-31 & $11: 00$ & $84 \%$ & 03:13:50 & \\
\hline 493-Jun-29 & $08: 37$ & $10 \%$ & 01:44:30 & \\
\hline \multicolumn{5}{|c|}{ Cuenta larga: $9 \cdot 3 \cdot 0.0 .0$} \\
\hline 500-Ago-10 & $18: 16$ & $63 \%$ & 00:49:12 & puesta de Sol eclipsado \\
\hline 502-Ene-24 & $08: 18$ & $61 \%$ & 02:19:55 & \\
\hline 504-May-29 & $11: 50$ & $30 \%$ & $02: 34: 29$ & \\
\hline 504-Nov-22 & $09: 30: 32$ & $14 \%$ & $02: 31: 25$ & \\
\hline 505-Nov-11 & $14: 23: 42$ & $59 \%$ & 03:08:28 & \\
\hline 507-Mar-29 & 05:54 & $15 \%$ & $00: 18: 47$ & salida de Sol eclipsado \\
\hline 511-Ene-15 & $06: 53: 41$ & $73 \%$ & $01: 27: 15$ & salida de Sol eclipsado \\
\hline \multicolumn{5}{|c|}{ Cuenta larga: $9 \cdot 4 \cdot 0.0 .0$} \\
\hline $515-\mathrm{Abr}-29$ & $16: 34: 44$ & $52 \%$ & $02: 26: 30$ & \\
\hline 523-May-30 & 12:11:42 & $23 \%$ & $02: 21: 17$ & \\
\hline 526-Mar-28 & $16: 12: 21$ & $89 \%$ & $02: 48: 36$ & \\
\hline $530-J u l-10$ & 09:03:09 & $39 \%$ & $02: 26: 08$ & \\
\hline \multicolumn{5}{|c|}{ Cuenta larga: $9 \cdot 5 \cdot 0.0 .0$} \\
\hline 537-Ago-21 & 05:44:11 & $65 \%$ & $01: 14: 40$ & salida de Sol eclipsado \\
\hline $537-$ Feb-25 & 09:45:37 & $2 \%$ & 01:03:02 & \\
\hline $544-0$ ct-02 & $10: 46: 59$ & $48 \%$ & $02: 57: 11$ & \\
\hline \multicolumn{5}{|c|}{ Cuenta larga: $9 \cdot 6 \cdot 0.0 .0$} \\
\hline $554-$ Sep-12 & $15: 53: 42$ & $72 \%$ & $02: 38: 25$ & \\
\hline $556-$ Feb-26 & 10:15:56 & $48 \%$ & $02: 26: 23$ & \\
\hline 559-Dic-14 & $16: 20: 06$ & $16 \%$ & 01:44:09 & \\
\hline $565-$ Feb-16 & $08: 57: 12$ & $97 \%$ & $02: 41: 54$ & \\
\hline 568-Dic-04 & 11:33:01 & $66 \%$ & 03:20:02 & \\
\hline 569-May-31 & $11: 01: 49$ & $55 \%$ & 03:20:27 & \\
\hline \multicolumn{5}{|c|}{ Cuenta larga: $9 \cdot 7 \cdot 0.0 .0$} \\
\hline 577-Jul-01 & 08:47:04 & $76 \%$ & $02: 42: 21$ & \\
\hline $580-A b r-29$ & $10: 45: 34$ & $69 \%$ & 03:35:04 & \\
\hline $581-0$ ct-13 & $09: 16: 32$ & $1 \%$ & 01:01:01 & \\
\hline 584-Ago-11 & 07:20:05 & $87 \%$ & $02: 16: 50$ & \\
\hline 587-Dic-05 & $07: 34: 27$ & $55 \%$ & 02:00:12 & \\
\hline 591-Mar-30 & $10: 06: 38$ & $10 \%$ & $01: 32: 34$ & \\
\hline 592-Sep-11 & 05:57:17 & $18 \%$ & 00:59:57 & salida de Sol eclipsado \\
\hline \multicolumn{5}{|c|}{ Cuenta larga: $9 \cdot 8 \cdot 0.0 .0$} \\
\hline 596-Ene-05 & $06: 29$ & $36 \%$ & 00:45:15 & salida de Sol eclipsado \\
\hline $602-$ Feb-27 & $14: 36: 15$ & $43 \%$ & 02:44:36 & \\
\hline $608-0$ ct-14 & 14:11:55 & $49 \%$ & 03:05:33 & \\
\hline 609-0ct-03 & $12: 42: 54$ & $30 \%$ & 03:04:18 & \\
\hline 610-Mar-30 & $10: 16: 16$ & $34 \%$ & $02: 11: 21$ & \\
\hline \multicolumn{5}{|c|}{ Cuenta larga: $9 \cdot 9 \cdot 0.0 .0$} \\
\hline 619-Mar-21 & 09:18:18 & $39 \%$ & 02:20:02 & \\
\hline
\end{tabular}




\begin{tabular}{|c|c|c|c|c|}
\hline Fecha juliana & $\begin{array}{c}\text { Máximo del } \\
\text { eclipse } \\
\text { (Hora del eclipse) }\end{array}$ & $\begin{array}{l}\text { Magnitud } \\
\text { Eclipse } \\
\text { Observable }\end{array}$ & $\begin{array}{l}\text { Duración del eclipse } \\
\text { observable (Horas: } \\
\text { minutos: segundos) }\end{array}$ & Observaciones \\
\hline 621-Ago-22 & 18:09 & $48 \%$ & $00: 31: 22$ & puesta de Sol eclipsado \\
\hline 623-Ene-06 & 14:45:39 & $45 \%$ & $02: 39: 12$ & \\
\hline 624-Jun-21 & 07:08:52 & $80 \%$ & $02: 32: 34$ & \\
\hline 630-Ago-13 & $18: 14$ & $83 \%$ & $00: 44: 01$ & puesta de Sol eclipsado \\
\hline 631-Ago-03 & $06: 24: 24$ & $76 \%$ & $01: 52: 50$ & salida de Sol eclipsado \\
\hline \multicolumn{5}{|c|}{ Cuenta larga: $9 \cdot 10.0 .0 .0$} \\
\hline 634-Jun-01 & $05: 49: 15$ & $63 \%$ & $01: 28: 45$ & salida de Sol eclipsado \\
\hline 635-Nov-15 & $09: 12: 53$ & $37 \%$ & $02: 59: 21$ & \\
\hline 638-Sep-13 & 07:09:38 & $87 \%$ & $02: 11: 25$ & \\
\hline 646-0ct-14 & $05: 50$ & $26 \%$ & $00: 36: 05$ & salida de Sol eclipsado \\
\hline $650-F e b-06$ & 07:29:37 & $95 \%$ & 02:24:01 & salida de Sol eclipsado \\
\hline 652-Dic-06 & $15 \cdot 50: 26$ & $7 \%$ & $01: 26: 25$ & \\
\hline 652-Jun-11 & 16:18:20 & $17 \%$ & 01:44:44 & \\
\hline \multicolumn{5}{|c|}{ Cuenta larga: 9.11.0.0.0 } \\
\hline 656-Mar-31 & 12:03:03 & $79 \%$ & 03:34:27 & \\
\hline 662-Nov-16 & $13 \cdot 55: 56$ & $50 \%$ & $03: 27: 28$ & \\
\hline 663-Nov-05 & $11: 43: 29$ & $29 \%$ & $03: 15: 37$ & \\
\hline 664-May-01 & $08: 41: 43$ & $72 \%$ & $02: 18: 35$ & \\
\hline 671-Jun-12 & $06: 37: 16$ & $28 \%$ & $01: 37: 53$ & \\
\hline \multicolumn{5}{|c|}{ Cuenta larga: $9.12 \cdot 0.0 .0$} \\
\hline 684-Sep-14 & $17: 52$ & $27 \%$ & 00:20:52 & puesta de Sol eclipsado \\
\hline 685-Sep-04 & $05: 44$ & $13 \%$ & $00: 20: 43$ & salida de Sol eclipsado \\
\hline 689-Dic-17 & $10-05: 38$ & $81 \%$ & $03: 54: 37$ & \\
\hline 689-Jun-22 & 16:58:10 & $16 \%$ & 01:23:12 & \\
\hline \multicolumn{5}{|c|}{ Cuenta larga: 9.13 .0 .0 .0} \\
\hline $692-0 c t-15$ & 08:27:06 & $24 \%$ & 01:51:18 & \\
\hline 696-Feb-08 & $15-20: 16$ & $64 \%$ & $02: 20: 31$ & \\
\hline 700-Nov-15 & $06-24: 02$ & $6 \%$ & $00: 54: 08$ & salida de Sol eclipsado \\
\hline 703-Sep-15 & $15: 55: 54$ & $5 \%$ & $01: 11: 42$ & \\
\hline 704-Mar-10 & $07: 41: 43$ & $75 \%$ & $02: 21: 25$ & \\
\hline 707-Ene-08 & $17: 29: 45$ & $76 \%$ & $01: 19: 24$ & \\
\hline 710-May-03 & $07: 30: 13$ & $91 \%$ & $02: 32: 57$ & \\
\hline $711-0 c t-16$ & $09: 36: 55$ & $49 \%$ & 02:29:40 & \\
\hline \multicolumn{5}{|c|}{ Cuenta larga: 9.14.0.0.0 } \\
\hline 713-Mar-01 & $06: 13$ & $26 \%$ & $00: 32: 50$ & salida de Sol eclipsado \\
\hline 716-Dic-18 & 14:29:54 & $33 \%$ & 03:07:08 & \\
\hline 717-Dic-07 & $12: 52: 40$ & $12 \%$ & $02: 57: 37$ & \\
\hline 718-Jun-03 & $06: 24: 35$ & $71 \%$ & 01:53:09 & \\
\hline 728-May-13 & 17:53:31 & $44 \%$ & 01:19:55 & puesta de Sol eclipsado \\
\hline \multicolumn{5}{|c|}{ Cuenta larga: $9.15 \cdot 0.0 .0$} \\
\hline 736-Jun-13 & $16-57: 24$ & $65 \%$ & 02:05:19 & \\
\hline 739-Abr-12 & $17: 17: 48$ & $81 \%$ & $02: 04: 16$ & puesta de Sol eclipsado \\
\hline 743-Jul-25 & 14:48:59 & $68 \%$ & $02: 23: 46$ & \\
\hline $750-$ Mar-12 & $16: 32: 33$ & $61 \%$ & 02:03:42 & \\
\hline $750-$ Sep-05 & 06:05:24 & $49 \%$ & 01:29:40 & salida de Sol eclipsado \\
\hline \multicolumn{5}{|c|}{ Cuenta larga: $9.16 \cdot 0.0 .0$} \\
\hline 751-Ago-25 & 10:58:29 & $7 \%$ & 02:04:19 & \\
\hline 757-Oct-17 & $16: 25: 32$ & $9 \%$ & $01: 27: 27$ & \\
\hline $758-\mathrm{Abr}-12$ & $06: 34: 23$ & $47 \%$ & $01: 49: 41$ & salida de Sol eclipsado \\
\hline
\end{tabular}




\begin{tabular}{|c|c|c|c|c|}
\hline Fecha juliana & $\begin{array}{l}\text { Máximo del eclipse } \\
\text { (Hora del eclipse) }\end{array}$ & $\begin{array}{l}\text { Magnitud } \\
\text { Eclipse } \\
\text { Observable }\end{array}$ & $\begin{array}{l}\text { Duración del eclipse } \\
\text { observable (Horas: } \\
\text { minutos: segundos) }\end{array}$ & Observaciones \\
\hline 761-Feb-09 & $17: 58$ & $74 \%$ & $00: 57: 17$ & puesta de Sol eclipsado \\
\hline 765-Nov-17 & 13:19:17 & $68 \%$ & 03:00:07 & \\
\hline 767-Abr-03 & $05: 49$ & $10 \%$ & $00: 20: 46$ & salida de Sol eclipsado \\
\hline 769-Mar-12 & $16: 59: 57$ & $4 \%$ & $00: 56: 12$ & \\
\hline \multicolumn{5}{|c|}{ Cuenta larga: $9.17 .0 .0 .0(771$-ene-20) } \\
\hline 771-Ene-20 & $14: 24: 28$ & $4 \%$ & $01: 38: 23$ & \\
\hline 772-Ene-09 & $14: 22: 16$ & $38 \%$ & 03:11:59 & \\
\hline 774-Nov-08 & $11: 27: 39$ & $7 \%$ & $01: 32: 56$ & \\
\hline $777-A b r-12$ & $05: 42$ & $18 \%$ & $00: 29: 20$ & salida de Sol eclipsado \\
\hline 778-Ago-26 & $17: 47: 27$ & $31 \%$ & $01: 08: 20$ & puesta de Sol eclipsado \\
\hline 783-Jun-04 & $14: 18: 31$ & $75 \%$ & $03: 34: 35$ & \\
\hline 786-Abr-03 & $05: 48$ & $31 \%$ & $00: 33: 16$ & salida de Sol eclipsado \\
\hline 790-Jul-16 & $13: 45: 59$ & $96 \%$ & 03:08:15 & \\
\hline \multicolumn{5}{|c|}{ Cuenta larga: $9 \cdot 18 \cdot 0.0 .0$} \\
\hline 793-May-14 & $12: 53: 37$ & $20 \%$ & $02: 31: 25$ & \\
\hline 793-Nov-08 & $06: 05: 14$ & $23 \%$ & $00: 54: 33$ & salida de Sol eclipsado \\
\hline 797-Ago-26 & $13: 32: 48$ & $97 \%$ & $02: 39: 55$ & \\
\hline 798-Feb-20 & 09:20:49 & $79 \%$ & 03:23:58 & \\
\hline 800-Dic-19 & $16: 38: 43$ & $3 \%$ & $00: 48: 50$ & \\
\hline $804-A b r-13$ & $16: 26: 07$ & $20 \%$ & $01: 35: 24$ & \\
\hline $805-$ Sep-26 & 09:08:51 & $42 \%$ & $02: 45: 51$ & \\
\hline \multicolumn{5}{|c|}{ Cuenta larga: $9.19 \cdot 0.0 .0$} \\
\hline 811-Nov-19 & $17: 22$ & $22 \%$ & $00: 28: 16$ & puesta de Sol eclipsado \\
\hline 815-Mar-14 & $17: 16: 27$ & $90 \%$ & $02: 01: 48$ & puesta de Sol eclipsado \\
\hline 819-Dic-20 & $16: 58: 49$ & $49 \%$ & $01: 31: 55$ & puesta de Sol eclipsado \\
\hline $823-A b r-14$ & $16: 19: 45$ & $33 \%$ & $01: 55: 09$ & \\
\hline $826-F e b-10$ & $14: 06: 21$ & $87 \%$ & 03:29:10 & \\
\hline 828-Dic-10 & 15:07:04 & $24 \%$ & $02: 02: 21$ & \\
\hline \multicolumn{5}{|c|}{ Cuenta larga: $10 \cdot 0 \cdot 0.0 .0$} \\
\hline $830-$ May-25 & 14:29:03 & $31 \%$ & $02: 27: 03$ & \\
\hline $832-\operatorname{Sep}-27$ & $17: 09: 58$ & $17 \%$ & $01: 17: 32$ & puesta de Sol eclipsado \\
\hline 837-Jul-06 & $06: 28: 59$ & $63 \%$ & $02: 16: 17$ & salida de Sol eclipsado \\
\hline 838 -Jun-25 & 10:46:11 & $5 \%$ & 01:42:06 & \\
\hline 840-May-05 & 05:29 & $5 \%$ & $00: 06: 23$ & salida de Sol eclipsado \\
\hline $842-0 c t-07$ & $15: 27: 36$ & $10 \%$ & $01: 25: 45$ & \\
\hline 844-Ago-17 & $10: 24: 06$ & $52 \%$ & $02: 59: 45$ & \\
\hline 847-Dic-11 & $07: 27: 16$ & $89 \%$ & $02: 27: 21$ & \\
\hline \multicolumn{5}{|c|}{ Cuenta larga: $10 \cdot 1 \cdot 0.0 .0$} \\
\hline $851-$ Sep-28 & 13:58:56 & $65 \%$ & 02:33:42 & \\
\hline 852-Mar-24 & $06: 27: 28$ & $40 \%$ & 01:40:08 & salida de Sol eclipsado \\
\hline 853-Mar-13 & $06: 53: 19$ & $10 \%$ & $01: 24: 58$ & \\
\hline 855-Jul-17 & $16: 31: 04$ & $54 \%$ & 02:23:07 & \\
\hline 856 -Ene-11 & $07: 38: 36$ & $60 \%$ & 02:15:17 & \\
\hline $859-0$ ct-29 & $09: 39: 33$ & $83 \%$ & 03:10:10 & \\
\hline 869-Abr-15 & 14:22:41 & $49 \%$ & 02:56:16 & \\
\hline \multicolumn{5}{|c|}{ Cuenta larga: $10 \cdot 2 \cdot 0.0 .0$} \\
\hline 877-May-16 & $14: 00: 04$ & $88 \%$ & $03: 02: 53$ & \\
\hline 880-Mar-14 & 11:54:14 & $69 \%$ & 03:35:18 & \\
\hline 881-Ago-28 & $06: 22: 28$ & $10 \%$ & $01: 24: 21$ & salida de Sol eclipsado \\
\hline
\end{tabular}




\begin{tabular}{|c|c|c|c|c|}
\hline Fecha juliana & $\begin{array}{l}\text { Máximo del eclipse } \\
\text { (Hora del eclipse) }\end{array}$ & $\begin{array}{l}\text { Magnitud } \\
\text { Eclipse } \\
\text { Observable }\end{array}$ & $\begin{array}{l}\text { Duración del eclipse } \\
\text { observable (Horas: } \\
\text { minutos: segundos) }\end{array}$ & Observaciones \\
\hline $884-J u n-26$ & 11:05:17 & $99 \%$ & 03:11:07 & \\
\hline \multicolumn{5}{|c|}{ Cuenta larga: $10.3 \cdot 0.0 .0$} \\
\hline 892-Jul-27 & 06:18:10 & $36 \%$ & 01:41:14 & salida de Sol eclipsado \\
\hline 898-Sep-19 & $08: 42: 45$ & $12 \%$ & $01: 46: 10$ & \\
\hline 899-Sep-08 & 17:11:26 & $59 \%$ & $01: 58: 32$ & puesta de Sol eclipsado \\
\hline 902-Ene-12 & 09:08:52 & $43 \%$ & 02:56:58 & \\
\hline $905-0$ ct-30 & $16: 22: 44$ & $47 \%$ & $02: 02: 59$ & \\
\hline $906-0 c t-20$ & $05: 52$ & $37 \%$ & $00: 26: 55$ & salida de Sol eclipsado \\
\hline \multicolumn{5}{|c|}{ Cuenta larga: $10 \cdot 4 \cdot 0.0 .0$} \\
\hline 909-Ago-18 & 11:06:25 & $7 \%$ & 02:05:02 & \\
\hline $910-$ Feb-12 & $10: 29: 35$ & $58 \%$ & $02: 47: 36$ & \\
\hline 913-Nov-30 & 12:38:51 & $26 \%$ & $02: 57: 12$ & \\
\hline 917-Mar-25 & 12:53:27 & $16 \%$ & 02:06:36 & \\
\hline 919-Feb-03 & 08:11:05 & $9 \%$ & $01: 26: 55$ & \\
\hline
\end{tabular}

Fuente: (Espenak, Five millennium catalog of solar eclipses, 2009)

\section{Eclipses de Luna}

En el período estudiado se identificaron 246 eclipses de luna totales y 309 eclipses parciales. Para los eclipses lunares totales se observa en la Tabla 5, la fecha juliana para cada eclipse, la hora de inicio y final del eclipse parcial, la hora de inicio y final del eclipse total y la duración del eclipse en horas, minutos y segundos. Asimismo, se detalla en la columna de observaciones, cuando la Luna aparece eclipsada durante la salida sobre el horizonte, 0 al contrario, cuando la Luna se pone en el horizonte durante la ocurrencia del eclipse, esta información esta relacionada con el tiempo de duración del eclipse, dado que no es visible el eclipse completo en la región de Copán (se presentan en color gris los datos de las horas donde el eclipse no es visible). A continuación, en la tabla 5 se detallan los eclipses lunares totales de la zona de Copán en el período de estudio dado: 


\begin{tabular}{|c|c|c|c|c|c|c|}
\hline Fecha juliana & $\begin{array}{c}\text { Inicio } \\
\text { Eclipse } \\
\text { Parcial }\end{array}$ & $\begin{array}{c}\text { Inicio } \\
\text { Eclipse Total }\end{array}$ & $\begin{array}{c}\text { Final } \\
\text { Eclipse Total }\end{array}$ & $\begin{array}{c}\text { Final } \\
\text { Eclipse } \\
\text { Parcial }\end{array}$ & Duración & Observaciones \\
\hline \multicolumn{7}{|c|}{ Cuenta Larga: 8.18 .0 .0 .0} \\
\hline 401-Dec-06 & $15: 43$ & $16: 54$ & $18: 16$ & $19: 26$ & 03:43 & Salida de Luna eclipsada \\
\hline 401-Jun-11 & $19: 45$ & $20: 46$ & $22: 19$ & $23: 20$ & 03:35 & \\
\hline 405-Sep-24 & 04:59 & 05:56 & 07:30 & $08: 27$ & 03:28 & Puesta de Luna eclipsada \\
\hline 408-Jan-28 & 17:06 & 18:17 & 19:09 & $20: 20$ & 03:14 & Salida de Luna eclipsada \\
\hline 408-Jul-23 & $17: 26$ & $18: 53$ & 19:35 & $21: 01$ & 03:35 & Salida de Luna eclipsada \\
\hline 409-Jul-12 & 19:16 & $20: 30$ & $21: 37$ & $22: 52$ & 03:36 & \\
\hline 412-May-12 & 04:44 & 05:42 & $07: 22$ & $08: 20$ & 03:36 & Puesta de Luna eclipsada \\
\hline \multicolumn{7}{|c|}{ Cuenta Larga: 8.19.0.0.0 } \\
\hline 416-Aug-24 & 03:03 & 04:11 & $05: 38$ & $06: 45$ & 03:42 & Puesta de Luna eclipsada \\
\hline 418-Dec-28 & 19:39 & $21: 03$ & $21: 37$ & $23: 00$ & 03:21 & \\
\hline 419-Dec-17 & $23: 55$ & 01:05 & $02: 28$ & 03:39 & $21: 39$ & \\
\hline Fecha juliana & $\begin{array}{c}\text { Inicio } \\
\text { Eclipse } \\
\text { Parcial }\end{array}$ & $\begin{array}{c}\text { Inicio } \\
\text { Eclipse Total }\end{array}$ & $\begin{array}{c}\text { Final } \\
\text { Eclipse Total }\end{array}$ & $\begin{array}{c}\text { Final } \\
\text { Eclipse } \\
\text { Parcial }\end{array}$ & Duración & Observaciones \\
\hline 419-Jun-23 & 03:03 & 04:06 & 05:31 & $06: 33$ & 03:30 & Puesta de Luna eclipsada \\
\hline 423-Apr-11 & $17: 24$ & $18: 30$ & $20: 10$ & $21: 16$ & 03:52 & Salida de Luna eclipsada \\
\hline 426-Feb-08 & 01:39 & 02:53 & 03:38 & $04: 52$ & 03:13 & \\
\hline 427-Jan-28 & $17: 36$ & $18: 38$ & $20: 00$ & $21: 01$ & 03:25 & Salida de Luna eclipsada \\
\hline 427-Jul-24 & 02:06 & 03:14 & 04:38 & 05:47 & 03:41 & Puesta de Luna eclipsada \\
\hline 430-Nov-15 & $20: 47$ & $21: 50$ & $23: 28$ & $00: 30$ & 03:43 & \\
\hline 434-Mar-10 & $20: 49$ & $21: 50$ & $23: 25$ & $00: 26$ & 03:37 & \\
\hline \multicolumn{7}{|c|}{ Cuenta Larga: 9.0.0.0.0 } \\
\hline 437-Jan-08 & 04:07 & 05:33 & 06:01 & $07: 28$ & 03:21 & Puesta de Luna eclipsada \\
\hline 438-Jun-23 & $02: 43$ & 03:55 & $04: 46$ & 05:57 & 03:14 & Puesta de Luna eclipsada \\
\hline 441-Apr-21 & 00:05 & 01:10 & $02: 55$ & 04:00 & 03:55 & \\
\hline 441-Oct-15 & $22: 02$ & $22: 59$ & $00: 35$ & $01: 32$ & 03:30 & \\
\hline 445-Feb-08 & 02:07 & 03:08 & $04: 33$ & 05:34 & 03:27 & \\
\hline 448-Jun-02 & $19: 26$ & $20: 25$ & $22: 02$ & 23:01 & 03:35 & \\
\hline 448-Nov-26 & 05:17 & $06: 19$ & 07:58 & $08: 59$ & 03:42 & Puesta de Luna eclipsada \\
\hline 452-Mar-21 & $04: 31$ & 05:31 & 07:09 & $08: 10$ & 03:39 & Puesta de Luna eclipsada \\
\hline 452-Sep-14 & $17: 51$ & $18: 56$ & $20: 34$ & $21: 39$ & 03:48 & Salida de Luna eclipsada \\
\hline 455-Jul-14 & $17: 53$ & 19:06 & $20: 00$ & $21: 12$ & 03:19 & Salida de Luna eclipsada \\
\hline \multicolumn{7}{|c|}{ Cuenta Larga: 9.1.0.0.0 } \\
\hline 456-Jan-08 & 16:08 & 17:18 & $18: 44$ & $19: 54$ & 03:46 & Salida de Luna eclipsada \\
\hline 463-Aug-14 & $16: 15$ & 17:18 & $18: 57$ & $20: 01$ & 03:46 & Salida de Luna eclipsada \\
\hline 466-Jun-14 & $02: 46$ & 03:47 & 05:17 & $06: 18$ & 03:32 & Puesta de Luna eclipsada \\
\hline 470-Sep-26 & 01:27 & 02:31 & 04:11 & $05: 16$ & 03:49 & \\
\hline 473-Jul-25 & 01:28 & 02:54 & 03:13 & $04: 40$ & 03:12 & \\
\hline 474-Jan-18 & $00: 06$ & 01:15 & 02:44 & 03:53 & 03:47 & \\
\hline 474-Jul-14 & $17: 46$ & $18: 47$ & $20: 09$ & $21: 10$ & 03:24 & Salida de Luna eclipsada \\
\hline \multicolumn{7}{|c|}{ Cuenta Larga: 9.2.0.0.0 } \\
\hline 477-Nov-06 & $15: 31$ & 16:28 & 18:05 & 19:02 & 03:31 & Salida de Luna eclipsada \\
\hline 481-Aug-24 & $23: 34$ & $00: 37$ & $02: 19$ & 03:21 & 03:47 & \\
\hline 481-Mar-01 & 18:45 & $19: 45$ & $21: 16$ & $22: 15$ & $03: 30$ & \\
\hline 484-Dec-17 & $22: 21$ & $23: 23$ & 01:01 & 02:03 & $03: 42$ & \\
\hline 488-Apr-11 & 19:37 & $20: 36$ & $22: 17$ & $23: 16$ & 03:39 & \\
\hline 492-Jul-25 & $01: 25$ & $02: 24$ & $03: 53$ & $04: 52$ & 03:27 & \\
\hline
\end{tabular}




\begin{tabular}{|c|c|c|c|c|c|c|}
\hline Fecha juliana & $\begin{array}{c}\text { Inicio } \\
\text { Eclipse } \\
\text { Parcial }\end{array}$ & $\begin{array}{c}\text { Inicio } \\
\text { Eclipse Total }\end{array}$ & $\begin{array}{c}\text { Final } \\
\text { Eclipse Total }\end{array}$ & $\begin{array}{c}\text { Final } \\
\text { Eclipse } \\
\text { Parcial }\end{array}$ & Duración & Observaciones \\
\hline 419-Jun-23 & 03:03 & 04:06 & 05:31 & $06: 33$ & 03:30 & Puesta de Luna eclipsada \\
\hline 423-Apr-11 & $17: 24$ & $18: 30$ & $20: 10$ & $21: 16$ & $03: 52$ & Salida de Luna eclipsada \\
\hline $426-F e b-08$ & 01:39 & $02: 53$ & 03:38 & $04: 52$ & 03:13 & \\
\hline 427-Jan-28 & $17: 36$ & 18:38 & $20: 00$ & $21: 01$ & $03: 25$ & Salida de Luna eclipsada \\
\hline 427-Jul-24 & $02: 06$ & 03:14 & $04: 38$ & $05: 47$ & 03:41 & Puesta de Luna eclipsada \\
\hline $430-$ Nov-15 & $20: 47$ & $21: 50$ & $23: 28$ & $00: 30$ & $03: 43$ & \\
\hline 434-Mar-10 & $20: 49$ & $21: 50$ & $23: 25$ & $00: 26$ & 03:37 & \\
\hline \multicolumn{7}{|c|}{ Cuenta Larga: 9.0.0.0.0 } \\
\hline 437-Jan-08 & 04:07 & 05:33 & 06:01 & $07: 28$ & 03:21 & Puesta de Luna eclipsada \\
\hline 438-Jun-23 & $02: 43$ & 03:55 & $04: 46$ & $05: 57$ & 03:14 & Puesta de Luna eclipsada \\
\hline 441-Apr-21 & $00: 05$ & $01: 10$ & $02: 55$ & 04:00 & 03:55 & \\
\hline $441-0 c t-15$ & $22: 02$ & $22: 59$ & $00: 35$ & $01: 32$ & 03:30 & \\
\hline $445-F e b-08$ & 02:07 & 03:08 & $04: 33$ & 05:34 & $03: 27$ & \\
\hline 448-Jun-02 & $19: 26$ & $20: 25$ & $22: 02$ & 23:01 & 03:35 & \\
\hline 448-Nov-26 & 05:17 & $06: 19$ & $07: 58$ & 08:59 & 03:42 & Puesta de Luna eclipsada \\
\hline 452-Mar-21 & $04: 31$ & $05: 31$ & 07:09 & 08:10 & 03:39 & Puesta de Luna eclipsada \\
\hline 452-Sep-14 & $17: 51$ & $18: 56$ & $20: 34$ & $21: 39$ & $03: 48$ & Salida de Luna eclipsada \\
\hline 455-Jul-14 & $17: 53$ & $19: 06$ & $20: 00$ & $21: 12$ & 03:19 & Salida de Luna eclipsada \\
\hline \multicolumn{7}{|c|}{ Cuenta Larga: $9 \cdot 1 \cdot 0.0 .0$} \\
\hline 456-Jan-08 & $16: 08$ & $17: 18$ & $18: 44$ & $19: 54$ & $03: 46$ & Salida de Luna eclipsada \\
\hline 463-Aug-14 & $16: 15$ & $17: 18$ & $18: 57$ & $20: 01$ & 03:46 & Salida de Luna eclipsada \\
\hline 466-Jun-14 & $02: 46$ & 03:47 & 05:17 & $06: 18$ & 03:32 & Puesta de Luna eclipsada \\
\hline $470-$ Sep-26 & $01: 27$ & $02: 31$ & $04: 11$ & $05: 16$ & $03: 49$ & \\
\hline 473-Jul-25 & $01: 28$ & $02: 54$ & 03:13 & $04: 40$ & 03:12 & \\
\hline 474-Jan-18 & $00: 06$ & $01: 15$ & $02: 44$ & 03:53 & 03:47 & \\
\hline 474-Jul-14 & $17: 46$ & $18: 47$ & $20: 09$ & $21: 10$ & 03:24 & Salida de Luna eclipsada \\
\hline \multicolumn{7}{|c|}{ Cuenta Larga: $9 \cdot 2 \cdot 0.0 .0$} \\
\hline 477-Nov-06 & $15: 31$ & $16: 28$ & $18: 05$ & $19: 02$ & 03:31 & Salida de Luna eclipsada \\
\hline 481-Aug-24 & $23: 34$ & 00:37 & 02:19 & 03:21 & 03:47 & \\
\hline 481-Mar-01 & $18: 45$ & $19: 45$ & $21: 16$ & $22: 15$ & 03:30 & \\
\hline 484-Dec-17 & $22: 21$ & $23: 23$ & 01:01 & $02: 03$ & $03: 42$ & \\
\hline 488-Apr-11 & 19:37 & $20: 36$ & $22: 17$ & $23: 16$ & 03:39 & \\
\hline 492-Jul-25 & $01: 25$ & $02: 24$ & $03: 53$ & $04: 52$ & $03: 27$ & \\
\hline \multicolumn{7}{|c|}{ Cuenta Larga: $9 \cdot 3 \cdot 0.0 .0$} \\
\hline 495-May-24 & $19: 36$ & $20: 43$ & $22: 21$ & $23: 28$ & 03.52 & \\
\hline 495-Nov-17 & $00: 23$ & 01:19 & $02: 56$ & 03:53 & 03:30 & \\
\hline 499-Mar-13 & $02: 51$ & 03:50 & $05: 25$ & $06: 23$ & 03:32 & Puesta de Luna eclipsada \\
\hline 502-Jul-05 & $17: 25$ & $18: 34$ & $19: 39$ & $20: 48$ & $03: 23$ & Salida de Luna eclipsada \\
\hline 503-Jun-25 & $03: 24$ & $04: 47$ & $05: 30$ & $06: 52$ & $03: 28$ & Puesta de Luna eclipsada \\
\hline 506-Apr-23 & 03:03 & 04:02 & $05: 43$ & $06: 42$ & 03:39 & Puesta de Luna eclipsada \\
\hline 506-Oct-17 & $17: 01$ & $18: 06$ & $19: 48$ & $20: 53$ & 03:52 & Salida de Luna eclipsada \\
\hline $510-F e b-09$ & $15: 38$ & $16: 46$ & 18:21 & $19: 28$ & $03: 50$ & Salida de Luna eclipsada \\
\hline 513-Jun-04 & $02: 03$ & 03:14 & $04: 40$ & $05: 51$ & $03: 48$ & Puesta de Luna eclipsada \\
\hline \multicolumn{7}{|c|}{ Cuenta Larga: 9.4.0.0.0 } \\
\hline 517-Sep-15 & $14: 44$ & $15: 45$ & $17: 29$ & $18: 30$ & 03:46 & Salida de Luna eclipsada \\
\hline 520-Jul-15 & $00: 47$ & 02:07 & $02: 46$ & 04:05 & 03:18 & \\
\hline 521-Jan-08 & $15: 22$ & $16: 23$ & $18: 02$ & $19: 03$ & $03: 41$ & Salida de Luna eclipsada \\
\hline 524-Oct-27 & $00: 58$ & $02: 03$ & 03:46 & $04: 50$ & 03:52 & \\
\hline 528-Aug-15 & 16.59 & $17: 56$ & $19: 33$ & $20: 30$ & 03:31 & Salida de Luna eclipsada \\
\hline $528-F e b-20$ & $23: 10$ & $00: 16$ & $01: 55$ & 03:01 & 03:51 & \\
\hline 531-Dec-09 & $18: 10$ & $19: 07$ & $20: 44$ & $21: 41$ & 03:31 & \\
\hline
\end{tabular}




\begin{tabular}{|c|c|c|c|c|c|c|}
\hline Fecha juliana & $\begin{array}{l}\text { Inicio } \\
\text { Eclipse } \\
\text { Parcial }\end{array}$ & $\begin{array}{c}\text { Inicio } \\
\text { Eclipse Total }\end{array}$ & $\begin{array}{c}\text { Final } \\
\text { Eclipse Total }\end{array}$ & $\begin{array}{c}\text { Final } \\
\text { Eclipse } \\
\text { Parcial }\end{array}$ & Duración & Observaciones \\
\hline \multicolumn{7}{|c|}{ Cuenta Larga: 9.5.0.0.0 } \\
\hline 535-Apr-03 & $18: 37$ & 19:34 & $21: 14$ & $22: 12$ & 03:35 & \\
\hline 535-Sep-26 & $22: 35$ & $23: 36$ & 01:19 & $02: 20$ & 03:45 & \\
\hline 539-Jan-19 & $23: 47$ & $00: 47$ & $02: 27$ & 03:27 & $03: 40$ & \\
\hline 539-Jul-16 & $16: 56$ & 18:04 & 19:30 & $20: 38$ & $03: 42$ & Salida de Luna eclipsada \\
\hline 542-May-14 & $17: 45$ & $18: 45$ & $20: 18$ & $21: 19$ & 03:34 & Salida de Luna eclipsada \\
\hline 546-Aug-27 & $00: 57$ & $01: 53$ & 03:32 & $04: 28$ & 03:31 & \\
\hline 546-Mar-03 & $06: 33$ & $07: 38$ & 09:20 & $10: 26$ & 03:53 & \\
\hline 549-Dec-20 & 03:04 & 04:01 & $05: 38$ & $06: 35$ & 03:31 & Puesta de Luna eclipsada \\
\hline 549-Jun-25 & $14: 58$ & $16: 34$ & $16-54$ & $18: 30$ & 03:32 & Salida de Luna eclipsada \\
\hline 550-Jun-14 & $16: 59$ & 18:11 & $19: 23$ & $20: 36$ & 03:37 & Salida de Luna edipsada \\
\hline 553-Apr-14 & $02: 18$ & 03:16 & $04: 56$ & $05: 54$ & 03:36 & Puesta de Luna eclipsada \\
\hline \multicolumn{7}{|c|}{ Cuenta Larga: $9 \cdot 6 \cdot 0.0 .0$} \\
\hline 557-Jul-26 & $23: 48$ & $00: 53$ & $02: 29$ & $03: 34$ & $03: 46$ & \\
\hline 560-May-25 & 01:04 & 02:07 & 03:31 & $04: 34$ & 03:30 & \\
\hline 560-Nov-18 & 17:07 & 18:11 & 19:55 & $21: 00$ & 03:53 & Salida de Luna eclipsada \\
\hline 568-Jun-24 & $23: 39$ & $00: 46$ & $02: 14$ & 03:21 & $03: 42$ & \\
\hline 571-Oct-18 & $14: 45$ & $15: 46$ & $17: 27$ & $18: 28$ & 03:43 & Salida de Luna eclipsada \\
\hline \multicolumn{7}{|c|}{ Cuenta Larga: 9.7.0.0.0 } \\
\hline $575-F e b-10$ & $16: 20$ & $17: 19$ & 19:00 & $20: 00$ & $03: 40$ & Salida de Luna eclipsada \\
\hline 578 -Nov-30 & 01:16 & $02: 20$ & 04:04 & 05:09 & 03:53 & \\
\hline 579-May-25 & $00: 45$ & $01: 56$ & $02: 48$ & 03:59 & 03:14 & \\
\hline 582-Mar-24 & $20: 48$ & $21: 53$ & 23:39 & $00: 44$ & 03:56 & \\
\hline $582-$ Sep-17 & $17: 16$ & 18:13 & 19:51 & $20: 47$ & 03:31 & Salida de Luna eclipsada \\
\hline 586-Jan-10 & $20: 43$ & $21: 40$ & $23: 18$ & $00: 15$ & 03:32 & \\
\hline 589-May-05 & $17: 20$ & 18:19 & 19:55 & $20: 54$ & 03:34 & Salida de Luna eclipsada \\
\hline $589-0 c t-28$ & $23: 02$ & $00: 04$ & $01: 43$ & $02: 45$ & $03: 43$ & \\
\hline $593-F e b-20$ & $00: 27$ & $01: 26$ & 03:07 & 04:07 & 03:40 & \\
\hline \multicolumn{7}{|c|}{ Cuenta Larga: 9.8.0.0.0 } \\
\hline 596-Jun-15 & $15: 45$ & $16: 59$ & $17: 49$ & $19: 03$ & 03:18 & Salida de Luna eclipsada \\
\hline 600-Apr-04 & $03: 42$ & $04: 47$ & $06: 33$ & $07: 38$ & 03:56 & Puesta de Luna eclipsada \\
\hline $600-$ Sep-28 & $01: 38$ & $02: 35$ & $04: 12$ & 05:09 & 03:31 & \\
\hline 604-Jan-22 & $05: 25$ & $06: 22$ & 08:00 & $08: 57$ & 03:32 & Puesta de Luna eclipsada \\
\hline 607-May-16 & $00: 43$ & $01: 44$ & 03:13 & $04: 15$ & 03:32 & \\
\hline 611-Aug-28 & $20: 58$ & $22: 01$ & $23: 46$ & $00: 50$ & 03.52 & \\
\hline \multicolumn{7}{|c|}{ Cuenta Larga: 9.9.0.0.0 } \\
\hline 614-Dec-21 & $17: 32$ & 18:37 & $20: 22$ & $21: 26$ & 03:54 & \\
\hline 615-Jun-16 & $15: 39$ & $16: 40$ & $18: 03$ & 19:04 & 03:25 & Salida de Luna eclipsada \\
\hline 622-Jul-27 & $20: 17$ & $21: 19$ & 23:03 & $00: 05$ & $03: 48$ & \\
\hline $625-$ Nov-19 & $15: 57$ & $16: 59$ & 18:37 & 19:38 & $03: 41$ & Salida de Luna eclipsada \\
\hline 629-Mar-14 & 16:19 & $17: 18$ & 18:59 & 19:58 & 03:39 & Salida de Luna edipsada \\
\hline 629-Sep-08 & $04: 16$ & 05:20 & 07:04 & 08:08 & 03:52 & Puesta de Luna eclipsada \\
\hline 633-Jan-01 & $01: 38$ & $02: 43$ & $04: 27$ & $05: 32$ & 03:54 & \\
\hline \multicolumn{7}{|c|}{ Cuenta Larga: $9 \cdot 10 \cdot 0.0 .0$} \\
\hline 633-Jun-26 & $23: 08$ & $00: 06$ & $01: 38$ & $02: 36$ & $03-28$ & \\
\hline 636-Apr-25 & $17: 10$ & 18:18 & 19:54 & $21: 02$ & 03:52 & Salida de Luna eclipsada \\
\hline 636-0ct-19 & $18: 42$ & $19: 40$ & $21: 16$ & $22: 13$ & 03:31 & \\
\hline 640 -Aug- 07 & 03:26 & $04: 28$ & $06: 11$ & $07: 13$ & $03: 47$ & \\
\hline $640-$ Feb-12 & $22: 28$ & $23: 25$ & $01: 05$ & $02: 02$ & 03:34 & \\
\hline 643-Jun-07 & $15: 20$ & $16: 31$ & $17: 32$ & $18: 43$ & $03: 23$ & Salida de Luna eclipsada \\
\hline 643-Nov-30 & $00: 32$ & 01:33 & 03:11 & $04: 12$ & $03: 40$ & \\
\hline
\end{tabular}




\begin{tabular}{|c|c|c|c|c|c|c|}
\hline Fecha juliana & $\begin{array}{l}\text { Inicio } \\
\text { Eclipse } \\
\text { Parcial }\end{array}$ & $\begin{array}{c}\text { Inicio } \\
\text { Eclipse Total }\end{array}$ & $\begin{array}{c}\text { Final } \\
\text { Eclipse Total }\end{array}$ & $\begin{array}{c}\text { Final } \\
\text { Eclipse } \\
\text { Parcial }\end{array}$ & Duración & Observaciones \\
\hline 644-May-27 & 01:17 & 02:37 & $03: 26$ & $04: 46$ & $03: 29$ & \\
\hline 647-Mar-25 & $00: 05$ & 01:04 & $02: 43$ & 03:43 & 03:38 & \\
\hline \multicolumn{7}{|c|}{ Cuenta Larga: 9.11.0.0.0 } \\
\hline 654-May-06 & $23: 46$ & $00: 57$ & $02: 21$ & $03: 33$ & $03: 47$ & \\
\hline $654-0 c t-31$ & $03: 24$ & $04: 22$ & $05: 57$ & $06: 54$ & $03: 30$ & Puesta de Luna eclipsada \\
\hline 661-Jun-17 & $22: 38$ & $00: 03$ & $00: 28$ & $01: 53$ & 03:05 & \\
\hline $665-$ Sep-29 & 19:17 & $20: 22$ & $22: 03$ & $23: 08$ & 03:51 & \\
\hline 669-Jan-22 & $17: 32$ & 18:37 & $20: 22$ & $21: 27$ & $03: 55$ & Salida de Luna edipsada \\
\hline \multicolumn{7}{|c|}{ Cuenta Larga: 9.12.0.0.0 } \\
\hline 676 -Aug-28 & $18: 13$ & $19: 16$ & $20: 52$ & $21: 55$ & $03: 42$ & \\
\hline 676-Mar-05 & 15:00 & 15:58 & 17:37 & 18:35 & 03:35 & Salida de Luna edipsada \\
\hline 679-Dec-22 & $17: 45$ & $18: 46$ & $20: 23$ & $21: 24$ & 03:39 & \\
\hline 683-Apr-16 & $15: 20$ & $16: 22$ & 17:51 & 18:53 & 03:33 & Salida de Luna eclipsada \\
\hline $683-0 c t-11$ & 03:00 & 04:06 & $05: 43$ & $06: 50$ & 03:50 & Puesta de Luna eclipsada \\
\hline $687-$ Feb-03 & 01:19 & $02: 24$ & 04:09 & $05: 14$ & $03: 55$ & \\
\hline $687-$ Jul-29 & $21: 56$ & $22: 52$ & 00:31 & 01:27 & 03:31 & \\
\hline 690-Nov-21 & $21: 02$ & $21: 59$ & $23: 34$ & $00: 32$ & $03: 30$ & \\
\hline 691-May-17 & $14: 50$ & $16: 03$ & $17: 16$ & $18: 28$ & $03: 38$ & Salida de Luna eclipsada \\
\hline \multicolumn{7}{|c|}{ Cuenta Larga: $9 \cdot 13 \cdot 0.0 .0$} \\
\hline 694-Mar-16 & $23: 03$ & 00:01 & 01:39 & $02: 37$ & $03: 34$ & \\
\hline 694-Sep-09 & $01: 51$ & $02: 55$ & $04: 27$ & 05:31 & $03: 40$ & \\
\hline 698-Jan-02 & $02: 20$ & 03:21 & $04: 58$ & 05:59 & 03:39 & \\
\hline 698-Jun-28 & $21: 20$ & $22: 24$ & $00: 03$ & 01:07 & 03:47 & \\
\hline 701-Apr-26 & $22: 52$ & $23: 55$ & 01:16 & $02: 20$ & 03:28 & \\
\hline 702-Apr-16 & $14: 54$ & 16:12 & $16: 46$ & 18:04 & 03:10 & \\
\hline 705-Aug-09 & $05: 41$ & $06: 38$ & 08:16 & 09:13 & 03:32 & \\
\hline 708-Dec-02 & $05: 53$ & $06: 51$ & $08: 26$ & 09:24 & 03:31 & \\
\hline 709-May-27 & $21: 33$ & $22: 40$ & 00:09 & 01:16 & $03: 43$ & \\
\hline \multicolumn{7}{|c|}{ Cuenta Larga: $9 \cdot 14 \cdot 0 \cdot 0.0$} \\
\hline 716-Jul-09 & 04:03 & 05:06 & $06: 50$ & $07: 53$ & $03: 50$ & \\
\hline 719-May-08 & $06: 19$ & 07:27 & $08: 35$ & $09: 42$ & $03: 23$ & \\
\hline 719-Nov-01 & $18: 45$ & 19:52 & $21: 26$ & $22: 33$ & $03: 48$ & \\
\hline 720-Apr-26 & $22: 33$ & $23: 42$ & $00: 40$ & $01: 49$ & 03:16 & \\
\hline $723-F e b-24$ & $16: 27$ & $17: 33$ & 19:16 & $20: 21$ & 03:54 & \\
\hline 726-Dec-13 & $14: 46$ & $15: 45$ & 17:19 & 18:17 & 03:31 & \\
\hline 727-Jun-08 & 04:16 & $05: 20$ & $06: 58$ & 08:02 & 03:46 & \\
\hline 730-Apr-07 & $14: 42$ & $15: 42$ & 17:14 & 18:14 & 03:32 & \\
\hline $730-$ Sep-30 & $17: 39$ & $18: 46$ & 20:07 & $21: 14$ & $03: 35$ & \\
\hline \multicolumn{7}{|c|}{ Cuenta Larga: 9.15.0.0.0 } \\
\hline 731-Sep-20 & $05: 44$ & 07:04 & $07: 38$ & $08: 57$ & $03: 13$ & \\
\hline 734-Jan-23 & $19: 21$ & $20: 22$ & $21: 58$ & $22: 58$ & 03:37 & \\
\hline 737-Nov-12 & $02: 45$ & $03: 53$ & $05: 25$ & $06: 33$ & 03:48 & \\
\hline 738-May-08 & $06: 08$ & 07:11 & $08: 26$ & 09:29 & 03:21 & \\
\hline 741-Aug-30 & $21: 34$ & $22: 32$ & 00:04 & 01:03 & 03:27 & \\
\hline 741-Mar-06 & $23: 48$ & $00: 55$ & $02: 35$ & $03: 41$ & $03: 53$ & \\
\hline 744-Dec-23 & $23: 38$ & $00: 36$ & $02: 11$ & $03: 09$ & 03:31 & \\
\hline 748-Apr-17 & $22: 20$ & $23: 22$ & $00: 48$ & $01: 50$ & $03: 30$ & \\
\hline $748-0 c t-11$ & $01: 46$ & $02: 54$ & 04:11 & $05: 19$ & $03: 33$ & \\
\hline 749-Sep-30 & 14:01 & $15: 15$ & 16:03 & $17: 16$ & 03:15 & \\
\hline
\end{tabular}




\begin{tabular}{|c|c|c|c|c|c|c|}
\hline Fecha juliana & $\begin{array}{l}\text { Inicio } \\
\text { Eclipse } \\
\text { Parcial }\end{array}$ & $\begin{array}{c}\text { Inicio } \\
\text { Eclipse Total }\end{array}$ & $\begin{array}{c}\text { Final } \\
\text { Eclipse Total }\end{array}$ & $\begin{array}{c}\text { Final } \\
\text { Eclipse } \\
\text { Parcial }\end{array}$ & Duración & Observaciones \\
\hline \multicolumn{7}{|c|}{ Cuenta Larga: 9.16.0.0.0 } \\
\hline 752-Feb-04 & 03:44 & $04: 45$ & $06: 19$ & $07: 20$ & $03: 36$ & \\
\hline 752-Jul-30 & $17: 39$ & $18: 42$ & $20-26$ & $21: 30$ & $03-51$ & \\
\hline 759-Mar-18 & $06: 58$ & 08:06 & $09: 41$ & $10-50$ & $03-52$ & \\
\hline 759-Sep-11 & $05: 41$ & $06: 41$ & 08:09 & 09:08 & 03:27 & \\
\hline 763-Jun-29 & $17: 51$ & $18: 53$ & $20: 37$ & $21: 39$ & $03: 48$ & \\
\hline 766-Apr-29 & $05: 50$ & $06: 56$ & 08:11 & 09:17 & 03:27 & \\
\hline 767-Apr-18 & 15:58 & $17: 39$ & $17: 41$ & 19:21 & $03-23$ & \\
\hline $767-0 c t-11$ & $22: 27$ & $23: 37$ & $00: 33$ & $01: 44$ & 03:17 & \\
\hline 770 -Aug-10 & $00: 34$ & $01: 39$ & 03:19 & $04: 24$ & 03:50 & \\
\hline \multicolumn{7}{|c|}{ Cuenta Larga: 9.17.0.0.0 } \\
\hline 773-Dec-03 & 18:56 & $20: 05$ & $21: 35$ & $22: 44$ & 03:48 & \\
\hline 774-May-29 & $21: 08$ & $22: 06$ & 23:39 & $00: 37$ & 03:29 & \\
\hline 777-Mar-28 & 14:01 & $15: 12$ & 16:39 & $17: 49$ & 03:48 & \\
\hline 777-Sep-21 & 13:55 & $14: 56$ & $16: 19$ & $17: 20$ & 03:25 & \\
\hline 781-Jan-14 & $17: 11$ & 18:09 & $19: 43$ & $20: 42$ & 03:31 & \\
\hline 781-Jul-09 & $00: 45$ & $01: 47$ & 03-29 & 04:31 & 03:46 & \\
\hline 784-Nov-01 & $18: 27$ & $19: 36$ & $20: 47$ & $21: 56$ & $03-29$ & \\
\hline 785-Apr-28 & $22: 56$ & $00: 14$ & $01: 09$ & $02: 27$ & 03:31 & \\
\hline 788 -Feb-25 & $20: 11$ & $21: 13$ & $22: 43$ & $23: 44$ & 03:33 & \\
\hline \multicolumn{7}{|c|}{ Cuenta Larga: 9.18.0.0.0 } \\
\hline 791-Dec-15 & 03:02 & $04: 11$ & $05: 40$ & $06: 49$ & $03: 47$ & \\
\hline 792-Dec-03 & $02: 13$ & $03: 56$ & $03-59$ & $05: 42$ & $03-29$ & \\
\hline 792-Jun-09 & 04:37 & $05: 33$ & $07: 11$ & 08:07 & 03:30 & \\
\hline 795-Apr-08 & $20: 55$ & $22: 09$ & $23: 23$ & $00: 38$ & $03: 43$ & \\
\hline 795-Oct-02 & $22: 17$ & $23: 20$ & 00:38 & $01: 41$ & 03-24 & \\
\hline 796-Mar-27 & $22: 15$ & $23: 41$ & $00: 18$ & $01: 44$ & $03: 29$ & \\
\hline 799-Jan-26 & $01: 49$ & $02: 48$ & 04:22 & $05: 21$ & 03:32 & \\
\hline 802-May-20 & $20: 35$ & $22: 03$ & $22: 23$ & 23.50 & 03:15 & \\
\hline 802-Nov-13 & $02: 57$ & 04:06 & 05:15 & $06-25$ & $03-28$ & Puesta de Luna eclipsada \\
\hline $803-$ Nov-02 & $15: 41$ & $16: 48$ & $17: 52$ & $18: 59$ & 03:18 & Salida de Luna eclipsada \\
\hline 806-Mar-08 & $04: 13$ & $05: 16$ & $06: 42$ & $07: 44$ & 03:31 & Puesta de Luna eclipsada \\
\hline 806-Sep-01 & $14: 46$ & $15: 55$ & $17: 21$ & $18: 30$ & 03:44 & Salida de Luna eclipsada \\
\hline $807-F e b-25$ & $19: 20$ & $20: 42$ & $21: 07$ & 2229 & 03:09 & \\
\hline \multicolumn{7}{|c|}{ Cuenta Larga: $9 \cdot 19 \cdot 0.0$} \\
\hline 813-Apr-19 & 03:43 & $05: 06$ & $05: 56$ & $07: 19$ & 03:36 & Puesta de Luna eclipsada \\
\hline 814-Apr-08 & $05: 18$ & $06: 33$ & 07:37 & $08: 53$ & 03:35 & Puesta de Luna eclipsada \\
\hline 814-Oct-02 & 19:55 & $21: 11$ & $22-02$ & $23: 18$ & $03-23$ & \\
\hline $817-J u l-31$ & $14: 55$ & $16: 00$ & $17: 30$ & $18: 35$ & $03: 40$ & Salida de Luna eclipsada \\
\hline 821-Nov-12 & $00: 27$ & $01: 33$ & $02: 39$ & $03: 46$ & 03:19 & \\
\hline 824-Sep-11 & $22-03$ & $23: 16$ & $00: 32$ & $01: 45$ & $03: 42$ & \\
\hline 825-Aug-31 & $22: 06$ & $23: 33$ & $00: 13$ & $01: 40$ & 03:34 & \\
\hline 825-Mar-08 & 03:34 & $04: 48$ & 05:33 & $06: 46$ & 03:12 & Puesta de Luna eclipsada \\
\hline 828-Dec-24 & 18:31 & 20:03 & $20: 29$ & $22: 01$ & 03:30 & \\
\hline 828-Jan-05 & 19:09 & $20: 20$ & $21: 46$ & $22: 56$ & 03:47 & \\
\hline 828-Jun-30 & 19:35 & $20: 31$ & $22: 10$ & 23:07 & 03:32 & \\
\hline \multicolumn{7}{|c|}{ Cuenta Larga: $10 \cdot 0.0 .0$} \\
\hline $831-$ Oct-24 & $15: 21$ & $16: 26$ & $17: 37$ & $18: 42$ & $03-21$ & Salida de Luna eclipsada \\
\hline $832-0 c t-13$ & 04:04 & $05: 17$ & $06: 16$ & $07: 29$ & $03-25$ & Puesta de Luna eclipsada \\
\hline 835-Aug-11 & $22: 13$ & $23: 21$ & $00: 41$ & $01: 49$ & 03:36 & \\
\hline
\end{tabular}




\begin{tabular}{|c|c|c|c|c|c|c|}
\hline $835-F e b-16$ & 18:45 & $19: 45$ & $21: 15$ & $22: 15$ & $03: 30$ & \\
\hline Fecha juliana & $\begin{array}{l}\text { Inicio } \\
\text { Eclipse } \\
\text { Parcial }\end{array}$ & $\begin{array}{c}\text { Inicio } \\
\text { Eclipse Total }\end{array}$ & $\begin{array}{c}\text { Final } \\
\text { Eclipse Total }\end{array}$ & $\begin{array}{l}\text { Final } \\
\text { Eclipse } \\
\text { Parcial }\end{array}$ & Duración & Observaciones \\
\hline 838-Dec-04 & $20: 09$ & $21: 19$ & $22: 27$ & $23: 36$ & $03: 27$ & \\
\hline 839-May-31 & 19:11 & $20: 16$ & $21: 55$ & $22: 59$ & $03: 48$ & \\
\hline 842-Mar-29 & 19:59 & $21: 06$ & $22: 16$ & $23: 23$ & $03-24$ & \\
\hline 842-Sep-23 & $05: 29$ & $06: 46$ & $07: 50$ & 09:07 & 03:38 & Puesta de Luna eclipsada \\
\hline 843-Sep-12 & 05:14 & $06: 33$ & $07: 35$ & $08: 53$ & 03:39 & Puesta de Luna eclipsada \\
\hline 846-Jan-16 & 03:07 & $04: 18$ & $05: 42$ & $06: 53$ & 03:46 & Puesta de Luna eclipsada \\
\hline 846-Jul-12 & 03:07 & 04:04 & $05: 41$ & 06:38 & 03:31 & Puesta de Luna eclipsada \\
\hline 847-Jan-05 & $02: 38$ & 04:07 & $04: 40$ & 06:09 & 03:31 & \\
\hline $849-$ Nov-03 & $00: 01$ & 01:07 & $02: 15$ & 03:21 & 03:20 & \\
\hline \multicolumn{7}{|c|}{ Cuenta Larga: $10 \cdot 1 \cdot 0.0 .0$} \\
\hline 850-Apr-29 & 19:10 & $20: 16$ & $21: 48$ & $22: 54$ & $03: 44$ & \\
\hline $853-F e b-27$ & 03:00 & 04:02 & 05:28 & 06:30 & 03:30 & Puesta de Luna eclipsada \\
\hline 854-Aug-11 & 18:01 & $19: 13$ & $20-06$ & $21: 18$ & 03:17 & Salida de Luna eclipsada \\
\hline 856-Dec-15 & 04:50 & 05:59 & 07:07 & 08:16 & $03-26$ & Puesta de Luna eclipsada \\
\hline 857-Dec-04 & 18:10 & 19:16 & $20: 23$ & $21: 29$ & 03:19 & \\
\hline 857-Jun-11 & 01:49 & $02: 52$ & 04:37 & $05: 40$ & 03:51 & Puesta de Luna eclipsada \\
\hline 860-Apr-09 & 03:43 & $04: 54$ & $05: 52$ & 07:03 & 03:20 & Puesta de Luna eclipsada \\
\hline 861-Mar-29 & 19:38 & $20: 43$ & $21: 53$ & $22: 58$ & 03:20 & \\
\hline 868-May-10 & 02:02 & 03:06 & $04: 45$ & 05:49 & 03:47 & Puesta de Luna eclipsada \\
\hline 868-Nov-03 & $20: 40$ & $21: 52$ & $22: 57$ & $00: 09$ & & \\
\hline \multicolumn{7}{|c|}{ Cuenta Larga: $10 \cdot 2 \cdot 0.0 .0$} \\
\hline 872-Aug-22 & 01:47 & $02: 53$ & 04:01 & $05: 08$ & 03-21 & \\
\hline 874-Dec-26 & 13:29 & $14: 38$ & $15: 45$ & $16: 54$ & $03: 25$ & \\
\hline 875-Dec-16 & 03:04 & 04:09 & 05:17 & $06: 23$ & 03:19 & Puesta de Luna eclipsada \\
\hline 878-Oct-14 & $20: 43$ & $22: 12$ & $22: 47$ & $00: 15$ & 03:32 & \\
\hline 879-Apr-10 & 03:30 & $04: 31$ & $05-51$ & $06: 53$ & $03: 23$ & Puesta de Luna eclipsada \\
\hline $879-0 c t-03$ & $20: 02$ & $21: 14$ & $22: 35$ & $23: 47$ & 03:45 & \\
\hline 882-Aug-02 & 18:26 & $19: 26$ & $20: 51$ & $21: 52$ & $03-26$ & \\
\hline $882-F e b-06$ & $18: 42$ & 19:56 & $21: 12$ & 2226 & 03:44 & \\
\hline 883-Jan-26 & 18:40 & $20: 02$ & $20: 51$ & $22: 13$ & 03:33 & \\
\hline $885-$ Nov-25 & $17: 35$ & $18: 42$ & $19: 47$ & 20:54 & 03:19 & \\
\hline 886-Nov-15 & 05:06 & $06: 17$ & $07: 25$ & 08:35 & 03:29 & Puesta de Luna eclipsada \\
\hline 889-Mar-20 & 19:04 & $20: 10$ & $21: 24$ & $22: 30$ & $03: 26$ & \\
\hline \multicolumn{7}{|c|}{ Cuenta Larga: 10.3.0.0.0 } \\
\hline 889-Sep-12 & $21: 02$ & $22: 27$ & $22-56$ & $00: 22$ & $03: 20$ & \\
\hline 890-Mar-10 & 04:59 & $06: 25$ & $06: 58$ & $08: 24$ & $03-25$ & Puesta de Luna eclipsada \\
\hline 893-Jan-05 & $22: 07$ & $23: 16$ & $00: 23$ & $01: 32$ & $03-25$ & \\
\hline 893-Jul-02 & 15:05 & $16: 09$ & $17: 52$ & $18: 56$ & $03-51$ & Salida de Luna eclipsada \\
\hline 896-Oct-25 & 04:31 & $06: 12$ & $06-21$ & 08:01 & 03:30 & Puesta de Luna eclipsada \\
\hline 897-Oct-14 & 03:41 & $04: 51$ & 06:18 & $07: 28$ & $03: 47$ & Puesta de Luna eclipsada \\
\hline 900-Aug-13 & $02: 13$ & 03:16 & $04: 32$ & 05:35 & $03-22$ & \\
\hline $900-F e b-18$ & 02:18 & $03: 34$ & $04: 43$ & 06:00 & $03: 42$ & \\
\hline 901-Aug-02 & 16:54 & $18: 15$ & 18.52 & $20: 12$ & 03:18 & \\
\hline 901-Feb-06 & $02: 33$ & $03: 51$ & $04: 49$ & 06:07 & 03:34 & \\
\hline 903-Dec-07 & $02: 24$ & $03: 32$ & $04: 36$ & 05:44 & 03:20 & \\
\hline 904-May-31 & $15: 41$ & $16: 42$ & $18: 27$ & $19: 28$ & 03:47 & \\
\hline 904-Nov-25 & 13:35 & $14: 46$ & $15: 54$ & $17: 05$ & 03:30 & \\
\hline 907-Apr-01 & $02: 53$ & 04:04 & 05:06 & 06:16 & $03-23$ & \\
\hline 908-Sep-12 & $17: 44$ & $18: 45$ & $20: 09$ & $21: 10$ & $03-26$ & \\
\hline
\end{tabular}




\begin{tabular}{|l|c|c|c|c|c|c|}
\hline Fecha juliana & $\begin{array}{c}\text { Inicio } \\
\text { Eclipse } \\
\text { Parcial }\end{array}$ & $\begin{array}{c}\text { Inicio } \\
\text { Eclipse Total }\end{array}$ & $\begin{array}{c}\text { Final } \\
\text { Eclipse Total }\end{array}$ & $\begin{array}{c}\text { Final } \\
\text { Eclipse } \\
\text { Parcial }\end{array}$ & Duración & Observaciones \\
\hline \multicolumn{7}{|c|}{ Cuenta Larga: 10.4 .0 .0 .0} \\
\hline 911-Jan-17 & $06: 42$ & $07: 51$ & $08: 56$ & $10: 06$ & $03: 24$ & \\
\hline 911-Jul-13 & $21: 45$ & $22: 51$ & $00: 28$ & $01: 34$ & $03: 49$ & \\
\hline
\end{tabular}

Tabla 5: Eclipses de Luna totales del 400 al 926 d.C. en Copán

Fuente: (Espenak, Five millennium catalog of solar eclipses, 2009).

Se presentan en gris las horas que no es visible el eclipse en el sitio de Copán

\section{Discusión de resultados}

El análisis de la información nos presenta que durante este período no se observaron eclipses totales en el sitio de Copán, solo eclipses anulares y parciales. A partir de los datos de los eclipses anulares y hasta 65 eclipses parciales con una magnitud superior al $50 \%$, se demuestra que en el sitio de Copán se pudieron observar eclipses que es poco probable que pasaran desapercibidos por la población de esa zona. De los 246 eclipses lunares totales, 194 de ellos tuvieron una fase de totalidad completa, es decir, que se observó la Luna completamente eclipsada, mostrando el tono rojizo que toma en esta etapa.

El registro de eventos astronómicos en Copán ha sido tema de varios autores, comentando registros de eclipses en la escalinata jeroglíica, en las estelas y en los templos. Schele y Looper (1994) plantean que en el Templo 11 de Copan y en la Estela E de Quirigua existe el registro de un evento cercano a la fecha 9.17.0.0.0, que lo asocian a un eclipse solar. Ese eclipse tuvo una magnitud del $4 \%$, dato muy bajo para que sea un eclipse fácilmente evidente. Este registro pudiera haberse logrado a partir de la predicción de los fenómenos que realizaban los mayas, más que de la simple observación, dada la magnitud observada.

Los datos de este estudio permiten visualizar los eclipses que resultarían más interesantes para ser registrados en las inscripciones de la ciudad con su respectiva asociación al gobernante que regía el sitio de Copán. A partir de la información resulta inicialmente relevante la ocurrencia de los cinco eclipses anulares de Sol, los 31 eclipses solares parciales que presentan una magnitud observable de más del $75 \%$ y los 194 eclipses lunares totales.

\section{Los eclipses durante el reinado del Décimo Tercer Gobernante de Copán}

A partir de la información antes mencionada se puede describir la ocurrencia de eclipses durante un período determinado. Durante el período de reinado del Décimo tercer gobernante de Copán Ilamado Waxaklaju'n U B'aah K'awiil, del 4 de julio de 695 al 27 de abril de 738, se produjeron los siguientes eclipses: 
Eclipses de Sol: Se produjo un eclipse anular en la fecha 3 de agosto de 696 (9.13.4.8.4 13 k'an 12 ch'en), con una duración total de 4 horas y 6 min de totalidad. Para los eclipses parciales de Sol, ocurrieron 13 eclipses parciales, donde solamente 3 sobrepasan una magnitud de $75 \%$, y con un eclipse solar, 3 de mayo de 710 , con una parcialidad del $91 \%$.

Eclipses de Luna: En este período ocurrieron 19 eclipses lunares totales, cuya totalidad pudo ser observada completamente y 27 eclipses lunares parciales.

\section{Conclusiones}

Durante el período de análisis se identificó la ocurrencia de 160 eclipses solares y 555 eclipses lunares. En el caso de los eclipses solares, se encontró que no se produjeron eclipses solares totales, pero si se produjeron 5 eclipses anulares, con duración de la totalidad desde $2 \mathrm{~m} 15 \mathrm{~s}$ hasta $9 \mathrm{~m} 11 \mathrm{~s}$ y 155 eclipses solares parciales. Durante el período de Waxaklaju'n U B'aah K'awiil se produjo un eclipse solar anular y por lo menos 3 eclipses con más del $75 \%$ de magnitud observable.

Los eclipses lunares que se produjeron en este período incluyen 246 eclipses de luna totales, de los cuales 194 de ellos pudo ser observada la totalidad completa y 309 eclipses parciales. Durante el período de Waxaklaju'n U B'aah K'awiil se produjeron 19 eclipse lunares totales, que a partir de las horas de ocurrencia se puede definir que la totalidad se pudo observar completamente.

El registro de eventos astronómicos, como ser eclipses, puede ser buscado de forma intencional a partir de los datos definidos en el estudio y las inscripciones del sitio de Copán, con énfasis en identificar registros de eclipses solares anulares y eclipses lunares totales, en cada período y gobernante correspondiente.

\section{Bibliografía}

- Aveni, A. (2005). Observadores del cielo en el México antiguo.Austin: The University of Texas Press.

- Espenak, F. (27 de Agosto de 2009). Five millennium catalog of lunar eclipses. Obtenido de http://eclipse.gsfc.nasa.gov/LEcat5/LEcatalog.html

- Espenak, F. (31 de Agosto de 2009). Five millennium catalog of solar eclipses. Obtenido de http://eclipse.gsfc.nasa.gov/SEcat5/SEcatalog.html

- Martínez, V., Miralles, J. A., Marco, E., \& Galadí-Enriquez, D. (2005). Astronomía fundamental. Publicacions de la Universitat de Valencia. 
- Meeus, J. (1991). Astronomical Algorithms. Virginia: Willman-Bell.

- Rice, P. (2004). Time, Astronomy and the Cosmos. Austin: University of Texas Press.

- Ruggles, C. (2005). Ancient Astronomy, An Encyclopedia of Cosmologies and Myth. Santa Barbara: ABC-CLIO, Inc.

- Schele, L., \& Looper, M. (1994). The 9.17.0.0.0 Eclipse at Quirigua and Copán. Copan notes - Copan Archaeological Acropolis Project.

- Thompson, E. (2006). Historia y religión de los Mayas (decimotercera edición ed.). México: Siglo Veintiuno.

- William, C. P. (2006). Publicaciones del Observatorio Astronómico Nacional. Bogotá: Observatorio Astronómico Nacional. 